Manuscript Accepted in Journal of Experimental Psychology: General on January $11^{\text {th }}, 2016$

This article may not exactly replicate the final version published in the APA journal. It is not the copy of record.

Eyelid-Openness and Mouth Curvature Influence Perceived Intelligence Beyond Attractiveness Sean N. Talamas ${ }^{1}$, Kenneth I. Mavor ${ }^{1}$, John Axelsson ${ }^{2}$, Tina Sundelin ${ }^{3}$ and David I. Perrett ${ }^{1}$

${ }^{1}$ School of Psychology and Neuroscience, University of St Andrews

${ }^{2}$ Department of Clinical Neuroscience, Division for Psychology \& Osher Center for Integrative Medicine, Karolinska Institute

${ }^{3}$ Department of Psychology, Stockholm University

Word Count: 12,196

Key Words: eyelid openness; mouth curvature; intelligence perception; attractive; sleep restriction

Acknowledgements: We thank Martin Campbell and Iris J Holzleitner for their helpful comments, Dengke Xiao for the experimental interface, Anne Perrett for proofreading, and Maria Garke, Ida Svensson, Cecilia Dagerskog, Henrik Cheng, and Frida Bayard for their help in data collection.

Address for correspondence:

Sean N. Talamas,

University of St Andrews, School of Psychology and Neuroscience,

St Mary's Quad, South Street, St. Andrews, Fife, KY16 9JP

Email: $\underline{\text { st87@st-andrews.ac.uk }}$

Ph.: +44 1334 463044, Fax: +44 1334463042 


\begin{abstract}
Impression formation is profoundly influenced by facial attractiveness, but the existence of facial cues which affect judgments beyond such an 'attractiveness halo' may be underestimated. Since depression and tiredness adversely affect cognitive capacity, we reasoned that facial cues to mood (mouth curvature) and alertness (eyelid-openness) affect impressions of intellectual capacity. Over four studies we investigated the influence of these malleable facial cues on first impressions of intelligence. In Studies 1 and 2 we scrutinize the perceived intelligence and attractiveness ratings of images of 100 adults (aged 18-33) and 90 school-aged children (aged 517) respectively. Intelligence impression was partially mediated by attractiveness, but independent effects of eyelid-openness and subtle smiling were found that enhanced intelligence ratings independent of attractiveness. In Study 3 we digitally manipulated stimuli to have altered eyelid-openness or mouth curvature and found that each independent manipulation had an influence on perceptions of intelligence. In a final set of stimuli (Study 4) we explored changes in these cues before and after sleep restriction, to examine whether natural variations in these cues according to sleep condition can influence perceptions. In Studies 3 and 4 variations with increased eyelid-openness and mouth curvature were found to relate positively to intelligence ratings. These findings suggest potential overgeneralizations based on subtle facial cues that indicate mood and tiredness, both of which alter cognitive ability. These findings also have important implications for students who are directly influenced by expectations of ability and teachers who may form expectations based on initial perceptions of intelligence.
\end{abstract}


Eyelid-Openness and Mouth Curvature Influence Perceived Intelligence Beyond Attractiveness First impressions of intelligence are likely to have profound consequences in various social contexts including education and employment. Perceptions of intelligence are strongly related to attractiveness. Dion, Berscheid, and Walster (1972) argued that, "what is beautiful is good" (p.285). This physical attractiveness bias is a well-studied example of Thorndike's (1920) 'halo effect' which leads to consistent, but not necessarily accurate, positive personality judgments of physically attractive people. The existence of facial cues which affect judgments beyond such an 'attractiveness halo' may be underestimated. In many studies, impressions are assumed to be based on stable characteristic of faces (masculinity, symmetry, averageness), but we argue for a focus on more dynamic and malleable facial cues in impressions of intelligence. Dynamic cues to the valence of emotions are increasingly recognized as influencing attributions but, beyond valence, further cues may derive from general level of arousal, and in particular tiredness.

\section{The Impact of Tiredness}

Tiredness can change over a relatively short time scale, can change on the base of behavioral choice, and has been shown to significantly reduce cognitive performance (Lim \& Dinges, 2010; Pilcher \& Huffcutt, 1996; Thomas et al., 2000; Walker, 2009). Facial cues of sleep deprivation have been shown to affect perceptions of attractiveness and health negatively (Axelsson et al., 2010). Sleep-deprived individuals also look sadder than non-sleep-deprived individuals, with corners of the mouth pointing downwards (Sundelin et al., 2013). An upturned mouth curvature may thus reflect alertness, but tiredness may also be reflected in the more direct facial cue of eyelid-openness; those who are sleep deprived are likely to have less eyelidopenness than those that are well rested (Sundelin et al., 2013). Additionally, overgeneralization 
of the relationship between tiredness and impaired cognitive performance may mean that facial cues related to tiredness influence perceptions of intelligence beyond the effects of attractiveness.

Overgeneralizations can be defined as a logical fallacy that occurs when a conclusion about a group is drawn from an unrepresentative (too small or narrow) sample (Walton, 1999). Zebrowitz, Fellous, Mignault, and Andreoletti (2003) argued that overgeneralizations involve accurate trait impressions from certain faces (a small unrepresentative sample) being used for other faces that are similar to the unrepresentative sample. Zebrowitz et al. (2003) extend the overgeneralization hypothesis to the anomalous face overgeneralization in which faces resembling those that are "unfit" illicit negative responses. For instance, the perception of individuals with droopy eyelids as tired and unintelligent may be an overgeneralized response based on the well-known relationship between sleep and decreased cognitive performance (Pilcher \& Huffcutt, 1996; Thomas et al., 2000).

Eye size effects. In exploring eyelid effects, we need to consider eye size more broadly, and in particular the relationship between eye size and the phenomenon of neoteny (the attractiveness of more ‘baby-faced’ features; Guthrie, 1970). Cunningham’s (1986) multiple motives hypothesis of physical attractiveness suggests people are attracted to individuals who display a combination of youthful (neotenous), mature, and expressive facial features. One fixed feature of neoteny that is considered attractive in both females and males is large eyes (Cunningham, Barbee, \& Pike, 1990; Cunningham, 1986)

For our purposes it is critical to note that the measurements used in Cunningham's (1986) study do not differentiate between eye size and eyelid-openness, whereas that distinction is crucial to our hypotheses. Cunningham's (1986) measure of eye size is the vertical separation of 
eyelids relative to vertical height of the face (forehead to chin) multiplied by the average width of each eye relative to the width of the face. We propose a measure of eyelid-openness as a different cue to social judgments as well as a contribution to Cunningham's (1986) measure of eye size. Indeed, we argue that eye size and eyelid-openness may actually elicit distinct social impressions. Neotenous features such as large eyes and foreheads, and small chins and noses, may trigger a caring response and the perception of child-like traits including naivety as part of an infant schema (Zebrowitz \& Montepare, 1992), whereas eyelid-openness may convey alertness. Taken together, these arguments suggest there can be a conflict between perceived intelligence and perceived attractiveness based on these fixed structural cues to age: people with large eyes should be perceived as attractive, youthful, but potentially naïve and hence less intelligent. Since we are interested in the effects of eyelid-openness over and above the effects of fixed facial features, we adopt a new approach to measuring eyelid-openness that controls for any underlying effects of eye size.

Mouth curvature. Smiling is a malleable facial cue that enhances attractiveness (Conway et al., 2008) and increases the rewarding nature of facial attractiveness (O’Doherty et al., 2003). Desired traits are more frequently ascribed to people who smile over those who don't (Lau, 1982). While a large grin could be interpreted as a naïve gesture, we argue that a slight upturn in mouth curvature (i.e. a subtle smile) is likely to influence perceived intelligence in a number of ways. Subtle differences in individual anatomy and facial feature configuration could be reminiscent of emotional expressions. Such hints of expression in supposedly neutral faces are known to affect the attribution of personality traits (Borkenau \& Liebler, 1992; Kenny, Horner, Kashy, \& Chu, 1992; Kleisner, Chvatalova, \& Flegr, 2014; Said, Baron, \& Todorov, 2009; Sutherland et al., 2013; Zebrowitz, Kikuchi, \& Fellous, 2010). Someone who is smiling is likely 
to be perceived as more trustworthy (Oosterhof \& Todorov, 2008), approachable, engaged and willing to interact (Jones, Debruine, Little, Conway, \& Feinberg, 2006). Additionally, a sad mood has been shown to significantly reduce cognitive performance (Ellis, Thomas, \& Rodriguez, 1984). Consequently, subtle smiling may be a malleable facial cue that has a particularly salient role in enhancing perceived intelligence independent of attractiveness.

Subtle smiling, sometimes referred to as positive emotional valence, has shown to have a marked influence on impression formation more broadly. From behavioral studies and computer modeling, Oosterholf and Todorov (2008) introduced a two-dimensional model demonstrating an underlying structure for face evaluations based on two primary dimensions: valence (or trustworthiness) and dominance. That is, while many attributions (intelligence, trustworthiness, extraversion, neuroticism, agreeableness, etc.) can be made to faces, all of these attributions can be accounted for by just differing amounts of the two dimensions. While Todorov and colleagues (2008) worked with faces with supposedly neutral expressions, subtle shape differences in mouth curvature reminiscent of smiling and frowning exerted a profound influence on impression of trustworthiness. Findings suggest that judgments of trustworthiness stem from an overgeneralization made to emotional expressions that may signal hostile or friendly intentions, which can be related to whether the person should be avoided or approached. Specifically, the valence dimension accounted for most of the variance in a variety of trait judgments (63.3\%) which were mainly related to positive judgments such as whether a face is perceived as attractive or responsible. On the other hand, the dominance dimension is more sensitive to features signaling physical strength (e.g., masculinity and maturity of age) and related to judgments such as aggressiveness and confidence. Sutherland et al. (2013) replicated the two-dimensional valence by dominance model of social inferences and added a new "youthful-attractiveness" 
factor. The emergence of the new dimension may reflect more realistic yet less standardized stimuli (e.g., overt expressions, dramatic differences in head posture).

\section{Static and Dynamic Cues}

The dimensions of dominance and trustworthiness/valence are particularly interesting considering their relatedness to fixed (or static) and malleable (or dynamic) cues to trait attributions. Hehman, Flake, and Freeman (2015) discuss the differences in the influence of static and dynamic facial cues on the attributions related to dimensions of valence and dominance. Hehman et al. (2015) argue that dynamic facial cues are more likely to influence judgments of trustworthiness/valence, while evaluations of dominance/physical ability are more likely to rely on static facial cues.

Thus, judgments of intentions tend to be based on dynamic facial cues that can change in a short time scale due to the action of facial musculature. Evaluations of dominance were found to be more consistent and rely heavily on static facial cues that depend on underlying bone structure; hence, less likely to change across time (Hehman et al., 2015). For example, the degree to which a person is smiling and has eyes wide open can change quickly. The changes could influence perceived mood and tiredness accordingly and in turn alter perception of intelligence, given the well-known relationship between tiredness and reduced cognitive ability (Ohayon \& Vecchierini, 2005; Pilcher \& Huffcutt, 1996; Wickens, Hutchins, Laux, \& Sebok, 2015).

Much of the research investigating attractiveness and the 'attractiveness halo' has focused on the fixed or static structural facial traits of symmetry, averageness, and sexual dimorphism (Langlois \& Roggman, 1990; Perrett et al., 1998, 1999). A 'good genes' theory of mate selection argues that these fixed traits are attractive because of their relationship to health (Rhodes, 2006). Yet, a number of more malleable characteristics have also been recently been 
shown to affect impressions of attractiveness. For example, skin color may signify current health state and can change over relatively short timescales with marked effects on attractiveness (Stephen, Law Smith, Stirrat, \& Perrett, 2009). We argue here that subtle changes of mouth curvature and eyelid openness might also have important impact on both perceived attractiveness and intelligence.

\section{Overview of the studies}

Given the adverse effects of low mood and fatigue on cognitive capacity, we reasoned that changeable facial cues to mood and alertness may affect impressions of intellectual capacity independent of their influence on attractiveness. Our overall aim was to examine how malleable facial cues relate to perceived intelligence by focusing on the role of facial cues to mood and alertness. Since both low mood and tiredness can impair cognitive performance, we hypothesized that (malleable) cues to mood and tiredness might affect attributions of intelligence through overgeneralizations. Cues to low mood and low alertness may also have a detrimental impact on attractiveness and therefore such cues could influence attributions of intelligence indirectly through the reduction in attractiveness. Alternatively the same facial cues may affect impressions of intelligence directly and independently of attractiveness judgments. We were therefore interested in exploring the direct effects of malleable facial cues on intellectual attributions, as well as any indirect effects mediated through the impact on attractiveness.

We began by examining attributions made to adult faces in images chosen to depict a neutral state without any posed expression of emotion. The images employed were from a human photo reference agency and had no overt facial expressions. Since impression formation can have influence throughout the lifespan, we also replicated the experiment with images of children's faces with neutral expressions. We then digitally manipulated the mouth curvature and eyelid- 
openness of stimuli independently to control for any other facial cues or combination of facial cues that could be driving impressions.

Finally, to investigate the malleable nature of these facial cues and to support ecological validity of the digital manipulation findings, we ran a fourth study to determine how subtle changes in facial appearance might alter trait attributions in sleep restricted individuals. We compared pairs of images of the same individuals rested and after sleep restriction. We assumed that, on average, sleep restriction will increase the tiredness of participants and will affect mood adversely. We note that tiredness is a common phenomenon that most adults and children experience occasionally and may well affect some individuals often and adversely during their education or employment (e.g. Oginska \& Pokorski, 2006; Perkinson-Gloor, Lemola, \& Grob, 2013; Perkinson-gloor et al., 2015). Hence, it is likely that cues to tiredness, such as eyelidopenness and subtle frowning will affect attributions and quite possibly expectations of performance.

\section{Study 1 - Adult Faces}

To examine cues of perceived intelligence across a stimuli set of 100 facial images, participants were asked to rate facial images of adults with neutral expressions for attractiveness and perceived intelligence. Objective measurements of eyelid-openness and mouth curvature were calculated and validated to assess the influence of these cues to alertness and mood on perception of attractiveness and intelligence. We hypothesized that eyelid-openness and hints of positive affect (upturned mouth curvature ${ }^{1}$ ) would enhance attribution of both attractiveness and perceived intelligence. We analyzed whether the impact of these facial cues on perceived intelligence was mediated by attractiveness, or whether effects were independent of attractiveness. We also controlled for the fixed trait of eye size, and we expected that large eyes 
would enhance ratings of attractiveness (Cunningham, 1986) but not necessarily intelligence.

Further, we examined the effect of gender since this variable might affect perceived intelligence through biased expectations of male intelligence (Beloff, 1992; Byrd \& Stacey, 1993; Furnham, Reeves, \& Budhani, 2002).

\section{Method}

\section{Face Stimuli}

The stimuli were 100 Caucasian two-dimensional faces that included 50 males $\left(M_{\text {age }}=\right.$ 25.3, $S D=4.64)$ and 50 females $\left(M_{\text {age }}=23.2, S D=3.74\right)$. These were chosen as the most standardized (e.g. clean shaven, neutral head posture) faces from a commercial database (available at www.3d.sk) designed for use in media and gaming development. An equal number of participants in each gender were purposefully chosen, but age of stimuli was not a part of selection criteria (no particular age range used for exclusions). All photographs chosen for this study were taken with a standardized camera set-up and lighting conditions; individuals had their hair pulled back, did not wear make-up or jewelry, and were instructed to pose with a neutral facial expression.

All face images were manually delineated with 188 points (Tiddeman, Burt, \& Perrett, 2001). Points were placed on face feature landmarks (e.g. eye corners and at regular intervals along the contours of facial features). Face images were aligned in size and position based on left and right pupils. Images were then resized and cropped (1608 x 2584 pixels) so that an equal proportion of hair and neck was shown in each image.

Objective measurements of facial stimuli. The degree of eyelid-openness was examined by taking the vertical distance from the center of the pupil to the top eyelid and dividing it by the width of the eye inner canthus to outer canthus (Figure 1). This measure was 
used because as an eyelid closes with tiredness, it is the top eyelid that lowers, rather than both eyelids meeting in the center. Cunningham's (1986) measurements of eye size (average width of each eye relative to the width of face multiplied by the vertical separation of eyelids relative to vertical height of the face forehead to chin) were also calculated.

Lastly, despite images supposedly showing neutral facial expressions, measurements of mouth curvature were calculated by taking the average height of the right and left corners of the mouth, subtracting the height of the center of the mouth and dividing by the width of the mouth (Figure 2).

--- Figures 1 and 2 about here ---

Perceptual rating validation of objective measures. Perceptual ratings of smiling and eyelid-openness were collected to validate the physical facial measurements and determine observers' awareness of cues that could potentially influence the attribution of intelligence. With perceptual validation, future research could use measurements of eyelid-openness and mouth curvature in lieu of perceiver ratings.

A group of 19 own-ethnicity participants (10 female, 9 male, $M_{a g e}=27, S D=12.95$ ) were recruited in an online experiment which (along with the other online studies presented in this paper) was advertised via the Perception Lab website (www.perceptionlab.com) and Facebook page (www.facebook.com/PerceptionLabStAndrews). No course credit or compensation was allotted. Participants were asked to rate the stimulus faces for degree of eyelid-openness. Given previous work (Elfenbein \& Ambady, 2002, 2003) that has highlighted differences in ability to recognize emotions on own- and other-race faces, exclusions were made on the basis of participant ethnicity (four participants excluded). Ratings in this task were of a 
very specific facial feature, hence fewer participants were studied compared to later tasks with more global ratings of attractiveness and perceived intelligence.

Participants first previewed the images, with each face being displayed for one second. After becoming familiar with the stimuli, participants received these instructions: "focusing on the eyelids, please rate the degree of eyelid-openness in this face compared to other faces presented". Stimulus order was randomized. Ratings of eyelid-openness for each face were averaged across all participants and correlated with the objective face measurements of eyelidopenness. As expected, the perceptual ratings of eyelid-openness correlated highly with the physical measurements $(r(98)=0.78, p<0.001)$.

The measure of mouth curvature takes into account both positive and negative reflections of a subtle smile/frown. Therefore in a separate experiment, instead of getting evaluators to rate the degree of smiling on a scale from 1-7, we emphasized to evaluators the subtlety of expression and allowed evaluators to give a response which reflects both a downward or an upward mouth curvature. A total of 51 new Caucasian (11 non-Caucasian excluded) participants (39 females, 12 males, $M_{\text {age }}=25.6, S D=9.44$ ) were recruited in an online experiment titled "Rate smiles in models". Participants viewed the same set of stimuli as above and were given the following instructions: "Please keep in mind that all the faces presented appear to have a neutral expression. None of these faces are meant to have obvious smiles. We are examining very subtle differences in mouth curvature." Evaluators were then presented with a face and asked "focusing on the mouth, please rate the degree of mouth curvature in this face compared to the other faces presented". Evaluators were able to use an unmarked sliding scale with end points labeled downward mouth curvature and upward mouth curvature; responses on the scale were recorded as an integer from $1-100$. Perceptual ratings of smiling significantly 
correlated with objective measurements of mouth curvature $(r(98)=0.77, p<0.001)$. Results provide evidence that subtle differences in smiling and frowning are perceivable. Indeed perceptual reports differentiate faces with a slight upturn or downturn in mouth curvature and show congruence with image measurements of mouth curvature.

\section{Participants}

Intelligence evaluators. After exclusions, a total of 173 participants (99 female, 74 male, $\left.M_{a g e}=28.26, S D=11\right)$ completed an online study entitled "Smart or Not". Participants assigning very similar ratings to all images (standard deviation of ratings $<0.50)$ were excluded (13 participants) due to their unusual and limited use of the scale which may indicate inadequate adherence to task instructions or the preconceived notion that social judgments like intelligence cannot be made from facial cues (or is socially undesirable). Further, participants who reported their ethnicity as different from 'white Caucasian' (35) were also excluded ${ }^{2}$, as stimuli presented were Caucasian and judgments of other ethnicities may be more susceptible to stereotypes (Zebrowitz, Bronstad, \& Lee, 2007). Participants were asked to rate 100 faces for perceived intelligence. Participants voluntarily gave informed consent and had to be over the age of 18 .

Attractiveness evaluators. After exclusions, an independent group of 140 participants (69 female, 71 male, $M_{\text {age }}=38.11, S D=10.41$ ) were recruited for an online study entitled "Adult Attractiveness" using Amazon Mechanical Turk and participated for payment to rate faces for perceived attractiveness. The same exclusion criteria used for intelligence evaluators was used for attractiveness evaluators. Two participants were excluded based on a standard deviation of ratings $<.50$ and 36 participants who reported their ethnicity as different from 'white Caucasian' were also excluded.

\section{Procedure}


Participants first completed a questionnaire inquiring about their age, gender, ethnicity, and country of origin. Next, participants previewed all stimuli with each image displayed for one second. The stimuli were then re-presented so that participants could rate the perceived intelligence of each face on a 7-point Likert-type scale with endpoints not at all intelligent and very intelligent. A separate group of evaluators rated the same stimuli on perceived attractiveness of each face on a similar 7-point scale with endpoints not at all attractive and very attractive. In both experiments, faces were presented in a random order. The minimum viewing time for each image was one second, but no maximum response time was enforced.

\section{Statistical analysis}

An average score of attractiveness and perceived intelligence was calculated for each of the 100 faces based on the average ratings across all participants. We expected a strong significant correlation between perceived attractiveness and perceived intelligence based on the attractiveness halo. Since our interest was in the impact of facial features on perceived intelligence beyond the attractiveness halo, we used mediation analyses to explore the direct effects of the measurements of eye size, eyelid-openness, and mouth curvature of each face on perceived intelligence and the indirect effects of these variables through perceived attractiveness while controlling for gender ${ }^{3}$. Given that Cunningham's (1986) measure of eye size is confounded with our measure of eyelid-openness, we expect these two measurements also to be strongly correlated. To see whether these measures have different relationships with perceptions of attractiveness and intelligence we examine models with the measurements of eyelid-openness and eye size included separately and with both measures in the mediation analysis, as we believe a more valid measure of eye size is one that controls for variation in eyelid-openness. 
An exploratory analysis of data was conducted to determine if the variables used were normally distributed. Results for the Kolmogorov-Smirnov test for normality indicated that measurements of mouth curvature and eyelid-openness, as well as averaged ratings of attractiveness and perceived intelligence did not deviate significantly from normal distribution. There were no problems with multicollinearity between variables (Variance Inflation Factor (VIF) for all variables $<1.5)$.

\section{Results}

\section{Facial averaging}

To help the reader visualize both the nature and subtlety of cues to perceived intelligence, differences are illustrated by facial averages of faces scoring low and high on perceived intelligence (Benson \& Perrett, 1992; Benson \& Perrett, 1993). Facial averages were constructed to examine the cues of perceived intelligence/attractiveness in male and female faces separately. Facial averages were synthesized from the top 20 male and female faces (10 male and 10 female faces) and bottom 20 male and female faces with the highest and lowest scores on perceived intelligence (Figure 3). This process (a) computes the average $\mathrm{x}$ and $\mathrm{y}$ values for 188 facial landmarks within the set of face images, (b) warps each shape of each facial image into these average coordinates, and then blends the warped component images (Re \& Perrett, 2012;

Tiddeman et al., 2001). These average images were then made symmetrical to emphasize the role of the cues being investigated (see Perrett et al., 1999).

--- Figure 3 about here ---

\section{Correlation Matrix}

After all the rating data had been collected, the main analysis can be done based on the ratings of the sample of 100 faces. As expected, there was a strong positive correlation between 
perceived attractiveness and perceived intelligence $(r(98)=0.72, p<0.001)$. Although we would expect a high correlation due to the attractiveness halo itself, this can also lead to concerns about the ability to distinguish these constructs empirically. We addressed this in two ways: (1) to correct the correlation for attenuation due to measurement error, and (2) to establish an indicator of discriminant validity. To address the issue of measurement error Cronbach's Alpha reliabilities were calculated for perceived intelligence (reliability $=0.98)$ and perceived attractiveness (reliability $=0.98)$. After correcting for attenuation, the correlation between attractiveness and intelligence ratings rises from $r=0.72$ to $r=0.73$, but this increase is not sufficient to undermine their distinctiveness. To establish discriminant validity the differences in correlations between the two physical measures (mouth curvature and eyelid-openness) and the two perceptual ratings (perceived attractiveness and intelligence) were investigated statistically using the Steiger (1980) test for dependent correlations. The correlation between attractiveness and eyelid-openness was significantly different from the correlation between intelligence and eyelid-openness $(r s=0.18,0.33 ; z=-2.06, p=.039)$. Similarly, the correlation between attractiveness and mouth curvature was significantly different from the correlation between intelligence and mouth curvature $(r s=0.04,0.24 ; z=-2.68, p=.007)$ supporting discriminant validity based on objective measures from the target faces. Taken together, these two analyses support the distinction between perceived attractiveness and intelligence measures.

As shown in Table 1, measurements of eyelid-openness and eye size were strongly correlated. There was no significant correlation between measurements of mouth curvature and measurements of eyelid-openness or measurements of eye size. There were also moderate correlations between gender of face and perceptual ratings indicating that female faces were perceived as more attractive and intelligent. However, gender was not significantly correlated 
with measurements of eyelid-openness, eye size, or mouth curvature. Older individuals tended to have upturned mouth curvature and reduced eye-size, but there were no significant correlation between age and eyelid-openness, attractiveness, or perceived intelligence ${ }^{4}$.

--- Table 1 about here ---

\section{Mediation Analysis}

To test whether perceived attractiveness mediates the relationship between measurements of eyelid-openness, eye size, mouth curvature, or gender on perceived intelligence, the SPSS plugin PROCESS was used (Hayes, 2013). Given the strong correlation between eye-openness and eye-size, we initially included these in two separate analyses to avoid potential suppression effects. We also included a third mediation analysis incorporating both variables.

The first mediation analysis conducted (see Table 2) examined perceived intelligence as the outcome variable, perceived attractiveness as the mediator, and measurements of eyelidopenness and mouth curvature as independent variables, with gender of face as a control variable. More eyelid-openness and female gender significantly predicted perceived attractiveness, but upturned mouth curvature was not a significant predictor (overall model: $R^{2}=$ $0.17, F(3,96)=6.47, p<0.001)$. Perceived attractiveness was a strong predictor of perceived intelligence, yet the direct effects of more eyelid-openness, upturned mouth curvature, and female gender significantly predicted perceived intelligence even with perceived attractiveness in the model (overall model: $R^{2}=0.63, F(4,95)=40.01, p<0.001$ ). Bias-corrected confidence intervals for indirect effects were calculated through 5000 bootstrap samples. The indirect effect (i.e. the mediation effect through perceived attractiveness) of measurements of eyelid-openness on perceived intelligence was not significant within the 95\% CI $(B=2.33, S E=1.36,95 \%$ CI [$0.22,5.14], \beta=0.13)$, but marginally significant within the $90 \% \mathrm{CI}(B=2.33, S E=1.36,90 \% \mathrm{CI}$ 
$[0.22,4.67], \beta=0.13)$. The indirect effect of measurements of mouth curvature on perceived intelligence through perceived attractiveness was not significant $(B=0.60, S E=0.92,95 \%$ CI [$1.31,2.36], \beta=0.04)$.

--- Table 2 about here ---

--- Figure 4 about here ---

The second mediation analysis (see Table 3) examined perceived intelligence as the outcome variable, perceived attractiveness as the mediator, and measurements of eye size (following Cunningham 1986) and mouth curvature as independent variables, with gender of face as a control variable. Female gender significantly predicted perceived attractiveness, but eye size and upturned mouth curvature were not significant predictors (overall model: $R^{2}=0.16$, $F(3,96)=6.12, p<0.001)$. Perceived attractiveness was a strong predictor of perceived intelligence, yet the direct effects of upturned mouth curvature and female gender significantly predicted perceived intelligence even with perceived attractiveness in the model. The direct effects of larger eye size did not significantly predict perceived intelligence (overall model: $R^{2}=$ $0.58, F(4,95)=32.81, p<0.001)$. The indirect effect of measurements of eye size on perceived intelligence (i.e. the mediation effect through perceived attractiveness) was found to be not significant $(B=31.21, S E=18.68,95 \% \mathrm{CI}[-0.96-72.44], \beta=0.12)$, nor was the indirect effect of measurements of mouth curvature on perceived intelligence through perceived attractiveness $(B=0.83, S E=0.98,95 \% \mathrm{CI}[-1.16,2.73], \beta=0.05)$.

--- Table 3 about here ---

The third mediation analysis (see Table 4) examined perceived intelligence as the outcome variable, perceived attractiveness as the mediator, and measurements of mouth curvature and both eyelid-openness and eye size as independent variables, with gender of face as 
a control variable. Female gender significantly predicted perceived attractiveness, but upturned mouth curvature, larger eye-size and more eyelid-openness were not significant predictors of perceived attractiveness (overall model: $R^{2}=0.17, F(4,95)=4.96, p=0.001$ ). Perceived attractiveness was a strong predictor of perceived intelligence, yet the direct effect of more eyelid-openness significantly predicted higher ratings of perceived intelligence, while there was a trend for the direct effect of larger eye size to be negatively associated with perceived intelligence with perceived attractiveness in the model. Upturned mouth curvature, and female gender also predicted perceived intelligence with perceived attractiveness in the model (overall model: $\left.R^{2}=0.64, F(5,94)=33.22, p<0.001\right)$. The indirect effect of measurements of eyelidopenness $(B=1.71, S E=1.67,95 \% \mathrm{CI}[-1.53,5.02], \beta=0.08)$, eye size $(B=14.93, S E=20.93$, $95 \% \mathrm{CI}[-24.86,59.43], \beta=0.05)$ and mouth curvature $(B=0.70, S E=0.95,95 \% \mathrm{CI}[-1.23$, 2.56], $\beta=0.05$ ) on perceived intelligence (i.e. the mediation effect through perceived attractiveness) were not significant.

--- Table 4 about here ---

\section{Discussion}

As expected, there were high correlations between attractiveness and perceived intelligence, reflecting the strength of the attractiveness halo. However, analyses taking into account the attenuation due to measurement error, and correlations demonstrating the discriminant validity of the two constructs support our argument that they can be considered as distinct constructs. This allows us to consider the impact of our other measures of interest on the attractiveness halo effect. Nonetheless, the high correlation does create potential for interpretative difficulties in regression, and under those circumstances we explicitly examine a mediation model that explores their relationship in a way that reflects the traditional 
understanding of the attractiveness halo. That is, we assumed that perceptions of attractiveness predict perceptions of intelligence, and we examined the direct and indirect effects of our focal and control measures on intelligence, potentially mediated by attractiveness.

The results of Study 1 are consistent with (based on a statistically non-significant trend) Cunningham's (1986) findings of large eyes being positively correlated with attractiveness. We argue that Cunningham's measure of eye size is a compound of two variables: the size of the eyes relative to the size of the face and the openness of eyelids. When examined conjointly in regression, neither measure of eye shape significantly predicted attractiveness, yet together they did explain a significant portion of variance in ratings of attractiveness ${ }^{5}$.

As highlighted by previous research, large eyes are a significant predictor of perceived baby-facedness, which has been shown to cause a baby face overgeneralization effect in which baby-faced individuals are perceived as more naïve (Zebrowitz \& Montepare, 1992). When controlling for eyelid-openness and perceived attractiveness in a linear regression model predicting intelligence, we found a non-significant trend for large eye size to negatively influence perceptions of intelligence.

In contrast, our new measure of eyelid-openness showed that more eyelid-openness increased perceived attractiveness and intelligence. Further, unlike the possible negative effect of large eye size on perceived intelligence, more eyelid-openness led to higher ratings of intelligence above and beyond the attractiveness halo. Likewise, while faces which had a subtly more positive mouth curvature were not perceived as any more attractive, an upturn in mouth curvature was related to a significant increase in perceived intelligence. Hence, the positive affect evident in the mouth configuration appears to have an impact on the attribution of intelligence independent of an attractiveness halo. 
These findings suggest that malleable or dynamic facial features have an impact on social attributions: people whose eyelids were more open were perceived as more intelligent than people who had more drooping eyelids. By the same token, people who had more of an upturned mouth curvature (i.e. showed a subtle smile) were not rated as more attractive, but were rated as more intelligent regardless of their attractiveness.

It should be noted that the faces used in this experiment have been used in other studies of social judgments (e.g. Batres \& Perrett, 2014; Quist, DeBruine, Little, \& Jones, 2012; Re \& Perrett, 2012). In all these experiments the faces were considered to show 'neutral' expressions. The differences in the malleable facial cues of eyelid-openness and upturn in mouth corners need not be based on an overt emotional expression; they could reflect individual differences in anatomy or subtleties in current mood and recent sleep patterns. Subtly upturned mouth curvature and increased eyelid-openness are more visible in the averages of faces judged high on intelligence compared to average of faces judged low on intelligence (see Figure 3).

Taken together, Study 1 highlights the influence, as well as the subtlety, of the malleable cues of eyelid-openness and mouth curvature on perceptions of intelligence above and beyond the effects of attractiveness. The findings have potential implications to environments in which perceptions of adult intelligence are crucial such as in business, law courts, and higher education. In each of these environments impressions of intelligence may influence expectations about competence. Given the special influence of perceptions of intelligence in education and the particular vulnerability of younger students to the expectancy effect (Alvidrez \& Weinstein, 1999; Rosenthal \& Jacobson, 1968), Study 2 set out to replicate findings using children as stimuli.

\section{Study 2 - Children's Faces}


In order to explore the generality of malleable facial cues on attributions we investigated the effect of eyelid-openness and mouth curvature on perceptions of intelligence (and attractiveness) in children's faces. As in Study 1, all faces showed neutral expressions. It was hypothesized that the influence of expressive features on attributions perceived in adult faces would affect children's faces equivalently. We thus anticipated that eyelid-openness and upturned mouth curvature would benefit attributions of intelligence beyond attractiveness in children's faces. Successfully replicating the findings in Study 1 across facial images of schoolaged children could have significant implications for educational contexts.

\section{Method}

\section{Face stimuli}

Stimuli consisted of children aged 5 to 16 with 49 boys $(M=9.38, S D=2.01)$ and 41 girls $(M=10.19, S D=2.75)$. Stimuli pictures were obtained from the Dartmouth Database of Children's Faces (Dalrymple, Gomez, \& Duchaine, 2013). All participants from the database that gave permission to be shared and presented were used as stimuli. All photographs were taken under standardized lighting conditions and camera set-up; individuals wore a black cap, did not wear make-up or jewelry, and posed with a neutral facial expression in front of a black background. As the black cap prevented accurate identification of the hairline, it was not possible to calculate Cunningham's (1986) measurements of eye-height ratio which takes into account the length of the face. Delineation, alignment, and cropping of the images followed the procedures outlined in Study 1.

Validation of perceptual ratings. A total of 16 new participants ( 15 female, 1 male, $\left.M_{a g e}=23.1, S D=10.9\right)$ were recruited in an online experiment. Seven non-Caucasian participants were excluded but no exclusions were necessary based on standard deviation (all 
within appropriate range). Participants followed the validation procedures outlined in Study 1. Mouth curvature and eyelid-openness ratings were assessed in two separate blocks. Block and stimulus order were randomized.

Ratings of mouth curvature and eyelid-openness for each face were averaged across all participants and correlated with the measurements of mouth curvature and measurements of eyelid-openness described in Study 1. The measurements of eyelid-openness and perceptual ratings of eyelid-openness were highly correlated $(r(88)=0.83, p<0.001)$. The perceptual ratings of mouth curvature also highly correlated with the objective measurements of mouth curvature $(r(88)=0.85, p<0.001)$.

\section{Participants}

Intelligence evaluators. After exclusions, a total of 76 new participants (45 female, 31 male, $\left.M_{\text {age }}=34.4, S D=13.90\right)$ were recruited for an online study entitled "How Smart Are These Kids". Participants were asked to rate children's faces for intelligence. Criteria identical to that in Study 1 were used to exclude participants from the final analysis: three excluded for standard deviation $<0.50$ and 14 excluded for not reporting ethnicity as 'white Caucasian'.

Attractiveness evaluators. After exclusions, an independent group of 60 participants (27 female, 33 male, $M_{\text {age }}=39.03, S D=11.26$ ) were recruited for an online study entitled "Children Attractiveness" using Amazon Mechanical Turk and participated for payment to rate faces for perceived attractiveness. The same exclusion criteria used for intelligence evaluators was used for attractiveness evaluators. No participants were excluded based on a standard deviation of ratings $<.50$ and 14 participants who reported their ethnicity as different from 'white Caucasian' were also excluded.

\section{Procedure}


As in Study 1 participants completed a questionnaire inquiring about their age, gender, ethnicity, and country of origin. Further, participants followed the procedures of Study 1 previewing stimuli at one second per face prior to the actual rating. The faces were then represented with participants using a 7-point Likert-type scale to rate faces for intelligence. An independent group of evaluators rated the stimuli for attractiveness using the same endpoints as those in Study 1. In each experiment, faces were presented in a random order with a minimum response time of one second and no maximum response time.

\section{Statistical Analysis}

An average score of attractiveness and perceived intelligence was calculated for each of the 90 faces based on the average ratings across all participants. Using similar methods following those used in Study 1, a mediation analysis was conducted to explore the direct and indirect effects of measurements of eyelid-openness and mouth curvature on perceptions of intelligence through, and independent of, attractiveness.

\section{Results}

\section{Facial averaging}

Again, face averaging processes outlined in Study 1 were used to synthesize 4 facial averages from the top 10 male and 10 female faces and the bottom 10 male and 10 female faces on rated intelligence (Figure 5). These composite images were then made symmetrical to make the investigated cues more salient.

---Figure 5 about here ---

\section{Correlation Matrix}

As expected, and shown in Table 5, there was a strong positive correlation between perceived attractiveness and perceived intelligence. There was also a significant correlation 
between gender and attractiveness such that female faces were perceived as more attractive and intelligent. Gender was not significantly correlated with measurements of eyelid-openness, eye size, or mouth curvature. Similar to findings from Study 1, there was no significant correlation between age and eyelid-openness, attractiveness, or perceived intelligence. Unlike the findings from Study 1, there was no correlation between age and mouth curvature. There was no significant correlation between measurements of mouth curvature and measurements of eyelidopenness.

--- Table 5 about here ---

\section{Mediation Analysis}

The mediation analysis (see Table 6) examined perceived intelligence as the outcome variable, perceived attractiveness as the mediator, and measurements of eyelid-openness and mouth curvature as independent variables, with gender of face as a control variable. More eyelidopenness and female gender significantly predicted perceived attractiveness, but upturned mouth curvature did not significantly predict perceived attractiveness (overall model: $R^{2}=0.15, F(3,86)$ $=5.25, p=0.002)$. Perceived attractiveness was a strong predictor of perceived intelligence, yet the direct effects of more eyelid-openness and upturned mouth curvature significantly predicted perceived intelligence with perceived attractiveness in the model. Direct effect of female gender was not significant in predicting perceived intelligence (overall model: $R^{2}=0.79, F(4,85)=$ 81.05, $p<0.001)$. Bias-corrected confidence intervals for indirect effects were calculated through 5000 bootstrap samples. The indirect effects of measurements of eyelid-openness $(B=$ $3.96, S E=1.79,95 \% \mathrm{CI}[0.50,7.71], \beta=0.19)$ on perceived intelligence through perceived attractiveness was significant, but mouth curvature was not $(B=1.79, S E=1.13,95 \%$ CI [-0.36, 4.11], $\beta=0.14)$. 
--- Table 6 about here ---

--- Figure 6 about here ---

\section{Discussion}

Results of Study 2 replicated the findings of Study 1 with children's faces. While in adult faces, perceived attractiveness may involve impression formation more related to sexual motives, previous literature investigating the perceived attractiveness of children in the bias towards kinship or self-similarity reveals that ratings of attractiveness does not necessarily imply sexual attraction, but rather the liking of another person (Park, Schaller, \& Van Vugt, 2008).

As in the adult faces, untrained participants were able to identify subtle differences in eyelid-openness and mouth curvature once their attention was drawn to the possible subtlety of the cues. Thus, objectively measured features were found to be congruent with perceptual ratings, validating the measures used. Further, congruent with findings in adult stimuli, upturn in mouth curvature did not significantly influence perceptions of attractiveness, but did predict increased ratings in perceived intelligence, while increased eyelid-openness had beneficial effects on both attractiveness and perceptions of intelligence. Similarly, as in Study 1 and predicted by the attractiveness halo effect, attractiveness ratings were significantly related to intelligence ratings. Hence, attractiveness mediated some of the beneficial effects of these cues on perceptions of intelligence. Yet, eyelid-openness and mouth curvature predicted ratings of intelligence independent of attractiveness. Thus, malleable facial cues to mood and alertness can have both direct and indirect impact on impressions of intellect.

Taken together, Studies 1 and 2 show that in both adults and children more eyelidopenness and upturned mouth curvature result in higher ratings of intelligence regardless of attractiveness. Most studies of facial attractiveness have investigated the impact of facial traits 
that are fixed (such as symmetry and averageness) or traits that can change only slowly (age, adiposity, skin texture, and color). Our analysis clearly showed that facial cues that are malleable over short timescales could also impact attributions. Yet because the cues of eyelid-openness and mouth curvature were investigated across different faces, it is possible that other facial features or a pattern of facial cues may be influencing perceptions of intelligence. Study 3 digitally manipulated stimuli to alter eyelid-openness and mouth curvature cues alone and investigated how these cues can independently influence perceptions of intelligence independent of other facial cues.

\section{Study 3 - Malleable Facial Cues Transform}

While Studies 1 and 2 found that the cues of eyelid-openness and mouth-curvature influence perceptions of intelligence across different sets of stimuli, this experiment was designed to investigate whether a targeted manipulation of these specific cues were sufficient to influence perceptions of intelligence. In order to isolate eyelid-openness and mouth curvature as independently predicting perceptions of intelligence, it is important to control for other facial features or a constellation of facial cues that may be influencing perceptions. Using stimuli from Study 2 we digitally manipulated the eyelid-openness and mouth curvature independently to examine whether differences in these cues alone would impact perceived intelligence.

\section{Method}

\section{Facial Stimuli}

Forty identities (20 female; $M_{\text {age }}=9.90, S D=2.74 ; 20$ male, $M_{\text {age }}=9.83, S D=2.13$ ) from the Dartmouth Database of Children's Faces (Dalrymple, Gomez, \& Duchaine, 2012) described in Study 2, were selected based on gender and similar age. Using Psychomorph, these 40 identities were transformed to create a total of 80 stimuli with differences in eyelid openness 
and mouth curvature. An average was made of the ten faces (out of the original 90 faces described in Study 2) with the most upturned/downturned mouth curvature and increased/decreased eyelid-openness respectively (irrespective of gender). The mouth shape and eye shape of these averages was used to transform the shape of individual faces. The first 20 randomly selected identities were transformed to create 40 stimuli (half of which were female) with different mouth curvature based on the upturned/downturned mouth curvature averages: 20 with an upturned mouth curvature, 20 with a downturned mouth curvature. A further 20 different identities were also transformed to create 40 stimuli with different eyelid-openness: 20 increased in eyelid-openness and 20 decreased in eyelid-openness (see Figure 7).

--- Figure 7 about here ---

\section{Participants}

After exclusions, a total of 37 new participants ( 7 males, 30 females; $M_{\text {age }} 21.65, S D=$ 5.12) were recruited to take part in a study entitled "Influences in the perception of intelligence in faces - in lab study" via SONA, an online research participation system for Psychology studies conducted at the University of St. Andrews and open to the community. Participants were compensated for their time at a rate of $£ 5 /$ hour pro rata. The same criteria as those in previous studies were used to exclude participants from the final analysis, with 23 participants excluded for not reporting ethnicity as 'white Caucasian' and no exclusions necessary for standard deviation $<0.50$.

\section{Procedure}

After completing a short questionnaire inquiring about their age, gender, ethnicity, and country of origin, participants rated the perceived intelligence of stimuli using a 7-point Likerttype scale with endpoints not at all intelligent and very intelligent. The 80 stimuli were arranged 
into two blocks, each with 10 upturned mouth curvature, 10 down turned mouth curvature, 10 increased eyelid-openness, and 10 decreased eyelid-openness. Each block contained 40 unique identities, i.e. no identity was repeated within one block so that each manipulation was done on a different identity and evaluators would not be presented with the same identity manipulated in different ways in the same block. Across the two blocks the only thing in the facial image to change was the upturn/downturn mouth curvature or the increase/decrease in eyelid-openness. Stimuli were standardized identical to that of Study 2: standardized lighting, black background, black caps, no make-up or jewelry, neutral expression and head posture.

\section{Results}

A one-way repeated measures ANOVA showed that the difference in intelligence ratings for stimuli with increased eyelid-openness $(M=4.15, S D=0.60)$ and decreased eyelid-openness conditions $(M=4.08, S D=0.51)$ was statistically significant, $F(1,35)=12.82, p=.001$, partial $\eta^{2}=.268$. Similarly, a one-way repeated measures ANOVA showed that the difference in intelligence ratings for stimuli with upturned mouth curvature $(M=3.98, S D=0.63)$ and downturned mouth curvature conditions $(M=3.79, S D=0.60)$ was statistically significant, $F$ $(1,35)=4.48, p=.042$, partial $\eta^{2}=.113$. There were no significant interactions for face gender with eyelid-openness $(F(1,35)=.204, p=.654)$ nor with mouth curvature $(F(1,35)=.761, p=$ $.389)$.

\section{Discussion}

The results of Study 3 emphasize the role of eyelid-openness and mouth curvature influencing perceptions of intelligence independent of any other fixed facial features or combination of facial features. Given that the facial transforms were made from natural variation in neutral resting expressions, it is evident that even the slightest differences in eyelid-openness 
and mouth curvature can significantly impact social judgments (see Figure 7). While the focus of this study was to emphasize the cues of eyelid-openness and mouth curvature influencing intelligence independently (rather than in combination with each other or with other fixed cues), it is possible (and likely) that these cues may also influence other social judgments, such as attractiveness. Study 1 and 2 found that differences in eyelid-openness and mouth curvature across individuals can influence perceptions of intelligence, while Study 3 found that when these cues alone (through digital manipulation) are changed within the same facial image there is an impact on perceived intelligence. The participant sample in this study was smaller (compared to Study 1 and Study 2), but the experimental design and the repeated measures statistical analysis shows that the cue manipulations were sufficient to drive judgments.

The findings of Study 3 are limited to single images of people and stimuli that have been digitally manipulated to change targeted cues. To confirm whether changes in appearance of real people have an impact on attributions, Study 4 compared different images of the same person and investigated whether a natural change in facial cues can lead to a change in trait attributions. That is, Study 4 attempts to improve the ecological validity and overall generalizability of findings by examining whether natural changes in eyelid-openness and mouth curvature within the same person alter perceived intelligence.

\section{Study 4 - Sleep-Restricted Faces}

To investigate the effect of malleable cues to perceived intelligence within the same stimuli we had participants rate the perceived intelligence and attractiveness of two facial images of the same person taken under separate conditions: after normal sleep and after sleep restriction. We expected that sleep restriction would be associated with negative mood manifested in a more downturned mouth configuration, and increased tiredness manifested in reduced eyelid-openness 
(Sundelin et al., 2013). We further predicted that changes in these facial features would impact perceptions of intelligence. A set of raters was then asked to choose which face had more eyelidopenness and was smiling more when comparing the two images of the same person taken under the two separate conditions. Hence, while Studies 1 and 2 showed that eyelid-openness and mouth curvature across individuals significantly influence perceptions, Study 4 investigated whether these effects are evident within the same person.

\section{Method}

\section{Face Stimuli Participants}

The stimuli pictures presented in this experiment came from the Sleep, Cognition, and Health lab at Karolinska Institutet in Stockholm, Sweden. Specifically, 25 individuals (14 females) were recruited and then photographed in two separate conditions: baseline and sleep restriction. A series of photographs were taken at each occasion with a standardized camera set up. Participants had their hair pulled back, no make-up or jewelry, and were asked to maintain a neutral facial expression. Five to eight representative photographs from each photo shoot were selected after low-quality photos (e.g. participant blinking) had been removed. A final selection "of the most representative photo" was done by an independent judge unaware of the experimental design or the sleep condition of the person in the photos. Their instructions were to choose the photo out of the five to eight available pictures that looked the most like the other photos taken at the same time. The individual judge did not know the participants in the photos. Todorov and Porter (Todorov \& Porter, 2014) highlight significant differences in person impressions within multiple facial photos of the same person due to random variation and discuss how this can influence accuracy of personality inferences based on faces. Thus, it was important to select the most standardized stimuli. 
These photographs were used for ratings in the second part of the study. The stimuli originally consisted of a total of 50 facial images from the two separate conditions (baseline and sleep-restricted), but one female was excluded prior to ratings for not having her hair properly combed back and another two female faces were excluded after ratings because of open mouths or exaggerated smiles (relative to the other stimuli), leaving the total number of stimuli at 44 facial images ( 11 females, 11 males, $M_{a g e}=22.9, S D=3.33$ ). We realize that this is a small sample and as such may limit interpretations, but given the unique contribution of this withinsubject study, we believe its contributions are still noteworthy.

Sleep-restriction procedures. Participants had a sleep need of seven to nine hours a night with no reported health problems or sleep disturbances. Participants (for stimuli) came into the lab on two separate occasions in a counter-balanced order; after spending at least eight hours a night in bed for two consecutive nights, and after spending no more than four hours a night in bed for two consecutive nights. The lab visits were at least one week apart. Adherence to the protocol was controlled via actigraphs (a small accelerometer worn on the non-dominant arm measuring activity and giving good information about sleep duration), sleep diaries, and text messages sent to the investigator at lights off and waking.

Validation of objective measures of feature changes. The purpose of this additional validation was to assess whether evaluators were capable of detecting a change in eyelidopenness and mouth curvature between sleep restriction conditions and whether these assessments matched objective measurements of eyelid-openness and mouth curvature. Studies 1 and 2 examined subtle differences in eyelid-openness and mouth curvature between different individuals. Study 4 enabled us to use pairs of facial images of the same individual to examine a more sensitive forced-choice paradigm to explore perceptions of these facial features. 
After exclusions, a total of 90 new participants (67 female, 23 male, $M_{\text {age }}=27.3, S D=$ 11.10) were recruited in an online study. Thirteen non-Caucasian participants and 22 participants who spent less than one second looking at an image were excluded. Participants completed a forced-choice experiment to compare the eyelid-openness and mouth curvature of the same person, between conditions. Specifically, participants were shown two images of the same face side-by-side, (baseline and sleep restricted) and were asked to "Please click on the face that is smiling more" or "Please click on the face that has its eyelids more open" in two separate blocks. Each participant completed both blocks ( 22 trials each block) and the order of blocks and stimulus faces was randomized.

Perceptual evaluations of eyelid-openness and smiling were calculated as the total count of observers choosing one face image over the corresponding face image from the alternative condition. This total count was then divided by the total number of observers (90 participants). This proportion defined perceptual evaluation of each face, which was then compared to the change in measurements of eyelid-openness and mouth curvature between conditions. In a partial correlation controlling for the age and gender of the face, the perceptual evaluation of eyelidopenness was positively correlated with the measured change in eyelid-openness $(r(20)=.62, p$ $=0.003)$ and the perceptual evaluation of smiling was significantly correlated with the measured change in mouth curvature $(r(20)=0.82, p<0.001)$ across the conditions. Thus, the malleable cues were indeed evident to observers and understood in a way that is consistent with the interpretation given to the objective measures.

\section{Attractiveness and Intelligence Raters}

After exclusions, a total of 61 participants ( 30 females, 30 males, 1 of unspecified gender, $\left.M_{\text {age }}=25.9, S D=6.10\right)$ rated 48 facial photographs with respect to attractiveness and 
intelligence. Participants were recruited at Universities and a work place in the Stockholm area and received a movie ticket for their participation. Participants who did not speak Swedish, were younger than 18 , older than 45 , or students of psychology were not recruited. No exclusions were necessary based on the same criteria used to exclude participants in previous studies (i.e. ethnicity and standard deviation of ratings).

\section{Procedure}

Participants rated 48 facial images for perceived intelligence and attractiveness. As part of a larger data collection, facial images were also rated for perceived tiredness, leadership ability, trustworthiness, and employability. The first four blocks of ratings were in a randomized order and consisted of intelligence, trustworthiness, leadership ability, and employability. After that, attractiveness and tiredness were rated, in that order. After a short test trial of four photos to familiarize the participants with the procedure, the photos were presented one-by-one and rated on 7-point Likert-type scales from 1 (not at all intelligent or very unattractive) to 7 (very intelligent or very attractive). The photographs were shown at a self-paced interval, for a maximum of six seconds. The presentations were made in a randomized order, with the exception that the two photographs from the same person (one from each condition) could not be presented back to back. After every 48th photograph, the participants performed a working memory task to prevent memorization of the faces and to avoid a familiarity effect.

\section{Statistical Analysis}

The ratings of attractiveness and intelligence for each face were averaged across raters and were then separated by condition. To examine the within-subject differences in eyelid-openness and mouth curvature pre- and post-sleep restriction, the change in ratings for the same face but different condition was computed (averaged baseline ratings subtracted by averaged sleep 
restricted ratings). This change of ratings between conditions was then compared to the measurements of change (baseline subtracted by sleep restriction) in eyelid-openness and mouth curvature across the 2 conditions. Gender was included as an individual level factor. To remain consistent with previous data analysis strategies, mediation analysis was conducted to examine the direct and indirect effects of the change in measurements of eyelid-openness and mouth curvature on change in perceived intelligence. However, because this is a within subject design with a small sample examining whether a change in malleable facial cues can change perceptions of intelligence within the same person, we were less concerned with whether this effect happened through or independent of the change in attractiveness and instead examined the total effects of these facial cues on the overall change in perceptions of intelligence.

\section{Results}

\section{Facial Averaging}

Again, described effects are visualized using the face averaging process explained in Study 1. Facial averages were synthesized from the top 10 male and female $(n=20)$ and the bottom 10 male and female $(n=20)$ faces; this time based on change in perceived intelligence (see Figure 8).

--- Figure 8 about here ---

\section{Sleep Condition}

Pre- and post-sleep restriction measurements of eyelid-openness and mouth curvature, along with ratings of attractiveness and perceived intelligence, were compared to investigate whether the condition of sleep restriction influenced any of these variables. Paired-samples ttests were used to compare measures and ratings across conditions (baseline vs. sleep restricted). For eyelid-openness, there was a significant difference in the scores for baseline $(\mathrm{M}=0.12, \mathrm{SD}=$ 
$0.02)$ and sleep restricted $(\mathrm{M}=0.11, \mathrm{SD}=0.02)$ conditions; $t(21)=3.16, p=0.005$. The mean difference in eyelid-openness was $0.01(95 \%[0.00,0.01])$ demonstrating a medium effect size, $\mathrm{d}$ $=0.67$. For mouth curvature, there was not a significant difference in the scores for baseline ( $\mathrm{M}$ $=0.00, \mathrm{SD}=0.03)$ and sleep restricted $(\mathrm{M}=-0.01, \mathrm{SD}=0.03)$ conditions; $(t(21)=1.43, p=$ 0.169. For attractiveness, there was a significant difference in the scores for baseline ( $M=3.62$, $\mathrm{SD}=0.76)$ and sleep restricted $(\mathrm{M}=3.48, \mathrm{SD}=0.82)$ conditions; $t(21)=2.70, p=0.013$. The mean difference in attractiveness was 0.14 (95\% [0.25, 0.33]) demonstrating a medium effect size, $d=0.58$. For perceived intelligence, there was a non-significant trend for a difference in the scores for baseline $(M=4.46, S D=0.59)$ and sleep restricted $(M=4.38, S D=0.61)$ conditions; $t(21)=1.82, p=0.083$. While the mean ratings did not differ significantly between conditions, it is possible, nonetheless, for changes in eyes and mouth to underlie changes in ratings across the two conditions

\section{Correlation Matrix}

As expected, there was a strong positive correlation between change in perceived attractiveness and change in perceived intelligence $(r(22)=0.75, p<0.001)$. There was no significant correlation between gender of face (female coded as 0 ) and average perceived attractiveness $(r(22)=-0.06, p=0.781)$ or perceived intelligence $(r(22)=-0.13, p=0.574)$. There was also no significant correlation between age of face and perceived attractiveness $(r(22)$ $=0.07, p=0.768)$ or perceived intelligence $(r(22)=-0.06, p=0.776)$. There was also no significant correlation between change in measurements of mouth curvature and change in measurements of eyelid-openness $(r(22)=0.10, p=0.666)$.

\section{Mediation Analysis}


The mediation analysis (see Table 7) examined change in perceived intelligence as the outcome variable, change in perceived attractiveness as the mediator, and change in measurements of eyelid-openness and measurements of mouth curvature as independent variables, with gender of face as a control variable (see footnote 3). Neither gender, change in more eyelid-openness, nor change in upturned mouth curvature were significant predictors of change in perceived attractiveness (overall model: $R^{2}=0.20, F(3,18)=1.46, p=0.258$ ). Change in perceived attractiveness was a strong predictor of change in perceived intelligence, yet the direct effects of change in upturned mouth curvature significantly predicted perceived intelligence with perceived attractiveness in the model. The direct effects of more eyelidopenness and gender were not significant predictors of change in perceived intelligence (overall model: $\left.R^{2}=0.76, F(4,17)=13.48, p<0.001\right)$. Bias-corrected confidence intervals for indirect effects were calculated through 5000 bootstrap samples. The indirect effects of change in measurements of eyelid-openness $(B=2.78, S E=2.71,95 \% \mathrm{CI}[-2.24,8.75], \beta=0.19)$ and mouth curvature $(B=1.37, S E=1.11,95 \% \mathrm{CI}[-0.68,3.78], \beta=0.19)$ on perceived intelligence through perceived attractiveness were both not significant.

However, the total effect of change in more eyelid-openness $(B=6.34, S E=3.01,95 \%$ CI $[0.12,12.67], p=0.050, \beta=0.36)$ and change in upturned mouth curvature $(B=4.79, S E=$ $1.32,95 \% \mathrm{CI}[2.02,7.56], p=0.002, \beta=0.60)$ on change in perceived intelligence was significant (overall model: $R^{2}=0.51, F(3,18)=6.33, p=0.004$ ).

--- Table 8 about here ---

--- Figure 9 about here ---

\section{Discussion}


Study 4 focused on how malleable cues influence perceived attractiveness and intelligence in the same person rather than across different individuals (Studies 1 and 2). The findings suggest that the malleable cues of smiling and eyelid-openness do influence perceptions of intelligence in the same person regardless of the effect of sleep condition on these cues. Interestingly, there was no significant relationship between change in eyelid-openness and smiling on perceived attractiveness, yet both malleable facial features had an influence on the change in perceived intelligence. The importance of smiling perception is apparent from the finding that images with a more upturned mouth curvature were perceived as more intelligent, independent of the impact of the attractiveness halo. Hence, regardless of fixed facial traits (such as averageness, symmetry, gender, and age), malleable cues can alter perceptions of intelligence.

Study 4 also examined the role of sleep restriction on attributions to faces and malleable facial features. It was found that sleep restriction was associated with people having less eyelidopenness, but sleep restriction was not associated with a change in mouth curvature. This gives objective support to the finding that sleep deprived people are perceived as having more heavy eyelids, but contrasts the finding that sleep deprived people are also judged to have more droopy mouth corners (Sundelin et al., 2013). We note that the previous study on this had a more severe form of sleep deprivation, and the degree of sleep deprivation may influence the effects on mouth curvature. Regardless, the change in mouth curvature from one photographic condition to another was significantly associated with perceptions of intelligence. That is, when comparing two images of the same person, the image with the more positive mouth curvature looks more intelligent. Thus, smiling is such a controllable cue that even people who are sleep deprived can be perceived as more intelligent (compared to a rested state) if they exhibit the subtlest upturn in mouth curvature. 
Taken together, the findings of Study 4 suggest eyelid-openness and mouth-curvature can subtly change within the same person and that these changes are perceivable and influence attributions of intelligence accordingly. Specifically, when a face had more eyelid-openness and more upturned mouth curvature, that face was perceived as more intelligent compared to an alternative facial image of the same person with less eyelid-openness and more downturned mouth curvature. Some fixed facial attributes such as averageness, sexual dimorphism, and skin pigment evenness affect facial attractiveness and may affect attribution of traits including intelligence. We have argued that malleable facial cues also have a role in attributions, but the degree of eyelid-openness and mouth curvature can also differ anatomically between individuals; one individual may on average have more droopy eyelids than another individual independent of the sleep habits of the two. All of these fixed traits may have a role in attributions made from facial appearance and could contribute to ratings in Studies 1 and 2. Studies 3 and 4 provide much more direct evidence that facial features changing within an individual can have effects on attributions of intelligence. Study 3 shows that changes in mouth curvature within individuals not only influence perceptions of attractiveness but also have independent effects on perceived intelligence.

\section{General Discussion}

The aim of the presented studies was to introduce and validate new measures to investigate malleable cues to perceptions of intelligence that are independent of the attractiveness halo. The cues of eyelid-openness and mouth curvature were targeted because of their likely relationship to sleep-deprivation and low mood, both of which have been associated with decreased cognitive ability (Ellis et al., 1984; Thomas et al., 2000). Our findings supported the hypothesis that more eyelid-openness and upturned mouth curvature increase ratings of 
perceived intelligence independent of attractiveness across adult faces (Study 1), children's faces (Study 2), and even within the same individual's face (Studies 3 and 4). We have focused on generality of the effect across ages and within the same person, and while we expect that the general point we are making would apply to other-race faces, we have limited our sample here for control purposes. The generality of the effects across races will need to be established in the future with a broader sample of faces and raters.

\section{Attractiveness}

Given the strength and pervasiveness of the attractiveness halo, it was important to determine whether the effects of the investigated cues stem from overgeneralizations of conditions which limit cognitive performance (i.e. alertness and mood) or only influence perceptions of intelligence through their impact on perceived attractiveness. It is clear from our findings that while the attractiveness halo is robust, there are certain malleable facial cues that can be changed to make it more likely that someone will be perceived as more or less intelligent regardless of their physical attractiveness.

\section{Overgeneralizations}

The beneficial impact of eyelid-openness and upturned mouth on perception of intelligence are likely to reflect overgeneralizations. Tiredness (Thomas et al., 2000; Walker, 2009) and low mood (Ellis et al., 1984) detract from current cognitive ability. Evaluators may therefore generalize from cues to tiredness and poor mood to the trait of intelligence; someone who looks awake and happy is thought to be more intelligent than someone who looks tired and unhappy. Overgeneralization from mouth shape to emotional state has been previously suggested to underlie social attributions (Oosterhof \& Todorov, 2008). Our findings suggest 
overgeneralizations arise from the eyes as well as the mouth. Moreover we show that both face cues are detected by observers and can be objectively measured in images.

\section{Mouth Curvature}

The shape of the mouth (expressing a subtle smile or frown) is a malleable facial cue that does not necessarily seem to influence perceived attractiveness across adult faces (Study 1) but does increase attractiveness in children's faces (Study 2) and significantly influences perceptions of intelligence in both adults (Study 1) and children (Study 2). It was also shown that a positive change in mouth curvature (more smiling) results in an increase in perceived intelligence ratings independent of other facial cues (Study 3) and within the same person (Studies 3 and 4).

While the mouth curvature is so subtle that at a first glance faces seem neutral (see Figures 3, 4, and 5), our findings suggest that observers are able to detect these subtle differences in mouth curvature and that these differences influence perceptions of intelligence accordingly. The reason smiling influences perception of intelligence above the attractiveness halo may be an overgeneralization effect related to sad mood and its effect in reducing cognitive performance (Ellis et al., 1984) or subtle frowning may indicate tiredness, which is related to worse cognitive functioning (Thomas et al., 2000; Walker, 2009).

More generally however, the finding that subtle cues may impact trait ratings has important implications. Oosterhof and Todorov (2008) argue that impressions of emotional valence in 'neutral' faces can affect trait judgments. While the stimuli faces in the current study did not seem to be smiling overtly or noticeably to the participants, a positive mouth curvature could enhance judgments of attractiveness and perceived intelligence and conversely subtle frowns could have adverse effects on trait evaluation. Further, it could be argued that unintentional smiles are a reflection of mood (i.e. a better mood might be accompanied by a 
subtle smile). Based on findings that a sad mood is associated with reduced cognitive performance (Ellis et al., 1984), signs of mood could potentially be a reflection of current cognitive capacity.

These results are important to consider in future studies where experimenters may assume facial stimuli to be 'neutral'. Differences in mouth curvature, which observers do not report as differences in smiling, seem to affect perceptions of intelligence. Indeed, measurements of mouth curvature may reveal effects on a variety of different social judgments of faces (i.e. perceived dominance, trustworthiness, and so on).

\section{Eyelid-Openness}

Eyelid-openness is another malleable facial cue that influences perceived intelligence independent of the attractiveness halo. The impact of eyelid-openness was observed both across faces of different people (Study 1 and 2) and within the face of the same person (Study 3 and 4). Observers validated the measurements used to calculate eyelid-openness variation across the faces of different people (adults and children) and within the face of the same person across two different conditions (Study 4).

It could be argued that although eyelid-openness is not fixed, one's ability to change eyelid-openness is less controllable than one's ability to alter mouth shape. While attempting to increase eyelid-openness (to look more alert or intelligent) is possible, it is difficult to do so without moving the eyebrows and looking surprised or unnatural. Perhaps unsurprisingly, results indicated that sleep restriction reduced eyelid-openness which was in turn detrimental to ratings of perceived intelligence.

We also found that in line with Cunningham's (1986) earlier findings, measurement of eye size correlated positively with ratings of attractiveness. However, when controlling for 
attractiveness and eyelid-openness, the neotenous fixed trait of large eyes is perceived as less intelligent, congruent with the baby-faced overgeneralization effect. Hence, while large eyes may positively influence attractiveness, large eyes relative to the size of the face can decrease rather than augment perceived intelligence. Further, the difference in direction between the effects of eyelid-openness and eye size on perceptions of attractiveness and intelligence points to the distinction between the perceived attractiveness and intelligence constructs despite their high correlations with each other.

\section{Gender}

Gender of the face was included as a factor in the analyses in order to control for any effects it may have on eye size and independent influences it may have on perceptions of intelligence. In adults' (Study 1) and children's (Study 2) faces, gender of the face significantly influenced perceptions of intelligence independent of attractiveness; females were judged to look more intelligent than males. These findings conflict with previous literature that finds females are perceived as less intelligent than males (e.g. (Beloff, 1992; Byrd \& Stacey, 1993). Specifically, Furnham and Rawles (1995) found that both men and women rate their fathers as having higher IQs than their mothers and rated their grandfather's IQ higher than their grandmother's IQ. Additionally, previous research has also highlighted that parents think their sons are brighter than their daughters (Furnham et al., 2002).

Such perceptions may be outdated as there is increasing evidence that women do better academically than men (Gurian \& Stevens, 2004). Gender was primarily included in our analyses as a control to the effects on the facial cues investigated. More research into the characteristics of the raters is needed to establish whether our findings represent a potential societal shift in perceptions of women's intelligence relative to men. 


\section{Conclusion and Implications}

We have documented two malleable facial cues that influence first impressions and may create an expectancy effect. We find that there is a strong relationship between the cue of eyelidopenness and perceptions of both attractiveness and intelligence, yet this cue has been largely overlooked in literature on social perception of faces. Further, we find mouth curvature in faces with a neutral expression has a pronounced influence on perceptions of intelligence. We show the effects of mouth curvature and eyelid-openness on facial perception is widespread, influencing intelligence judgments in adults and children alike.

The judgments of attractiveness and competence have a broad range of implications influencing perceptions of success (Rule \& Ambady, 2010), hiring and promotion (Rule \& Ambady, 2009), and mate-selection (Eagly, Ashmore, Makhijani, \& Longo, 1991; Moore, Filippou, \& Perrett, 2011). The implications for children are perhaps most important. Children arriving at school tired from inadequate sleep or in low mood due to adverse home life are likely to be judged as less intellectually able. In turn these judgments are likely to lower teacher expectations and hinder educational attainment (Rosenthal \& Jacobson, 1968). Yet, we show that the cues on which intelligence judgments hinge are changeable. Hence, improvements in sleep patterns and mood for any individual may benefit social evaluations and expectations over and above any impact on actual cognition. The subtlety of the cues studied here means that that the role of malleable facial features in society and their influence in diverse situations, such as in the formation of teacher expectations and employee recruitment, is likely to have been underestimated. 


\section{Notes}

1. The term mouth curvature is used throughout most of the paper instead of smiling or frowning because the faces observed were considered neutral; not overtly smiling or frowning. Thus, the subtle differences influencing perceptions are not based on an overt emotion, but rather the shape of the mouth.

2. Non-Caucasian raters were excluded, as is sometimes conventional in this area, to avoid the ambiguity of any cross-race effects on perceptions of attractiveness and intelligence. Including the non-Caucasian raters for every analysis reported did not change the pattern of results. In most cases, the effects were stronger with these raters included. Given the small sample of non-Caucasian raters, the generality of the effects across races will need to be established in the future with a broader sample of faces and raters.

3. The gender of the face was used as a control throughout all the studies because of its influence on perceived intelligence (unlike age). The patterns of findings do not change when gender of face is not including as a control.

4. The variable of age was explored additionally because of the potential effect age can have on eye size and perceptions of naivety. Age was not a significant predictor of perceived intelligence in children or adults, and is not included in models for simplicity. Including age in the models does not contribute to the model or change the pattern of results. It is possible that the effects of age on perceptions of intelligence were not significant because the samples in which this potential cue was examined were too narrow in age range. Future research would benefit from examining the effects of age on perceptions of intelligence in a larger and more varied sample. 
5. It may seem surprising that in the regression model neither eye size, nor eyelid-openness was a significant predictor of attractiveness in adults. The lack of significance of each variable as a predictor does not mean that these variables have no influence. The conjoint contribution of the two eye variables is demonstrable in a hierarchical regression model $\left(1^{\text {st }}\right.$ step independent variables: mouth curvature and sex of face, $2^{\text {nd }}$ step independent variables: Cunningham's eye size and eyelid openness). In the second stage the model showed a significant increase in variance explained by eye size and eyelid openness conjointly $\left(R^{2}\right.$ change $\left.=0.08 p=0.012\right)$. However, their high correlation means that their unique contributions are undermined when both are included in regressions. 


\section{References}

Alvidrez, J., \& Weinstein, R. S. (1999). Early teacher perceptions and later student academic achievement. Journal of Educational Psychology, 91(4), 731-746.

Axelsson, J., Sundelin, T., Ingre, M., Someren, E. J. W. Van, Olsson, A., \& Lekander, M. (2010). Beauty sleep: experimental study on the perceived health and attractiveness of sleep deprived people. British Medical Journal, 341(c6614), 1-5. doi:10.1136/bmj.c6614

Batres, C., \& Perrett, D. I. (2014). The influence of the digital divide on face preferences in El Salvador: People without internet access prefer more feminine men, more masculine women, and women with higher adiposity. PLoS ONE, 9(7). doi:10.1371/journal.pone.0100966

Beloff, H. (1992). Mother, father and me: Our IQ. The Psychologist, 5, 309-311.

Benson, P., \& Perrett, D. I. (1992). Face to face with the perfect image. New Scientist, 133(1809), 32-35.

Benson, P., \& Perrett, D. I. (1993). Extracting prototypical facial images from exemplars. Perception, 22, 257-262. doi:10.1068/p220257n

Borkenau, P., \& Liebler, A. (1992). Trait inferences: Sources of validity at zero acquaintance. Journal of Personality and Social Psychology, 62(4), 645-657.

Byrd, M., \& Stacey, B. (1993). Bias in IQ perception. The Psychologist, 6, 1-16.

Conway, C. A., Jones, B. C., Debruine, L. M., Little, A. C., Hay, J., Welling, L. L. M., ... Feinberg, D. R. (2008). Integrating physical and social cues when forming face preferences: differences among low and high-anxiety individuals. Social Neuroscience, 3(1), 89-95. doi:10.1080/17470910701676145 
Cunningham, M. R. (1986). Measuring the physical in physical attractiveness : Quasiexperiments on the sociobiology of female facial beauty. Journal of Personality and Social Psychology, 50(5), 925-935.

Cunningham, M. R., Barbee, A. P., \& Pike, C. L. (1990). What do women want? Facialmetric assessment of multiple motives in the perception of male facial physical attractiveness. Journal of Personality and Social Psychology, 59(1), 61-72.

Dalrymple, K. A., Gomez, J., \& Duchaine, B. (2013). The Dartmouth database of children's faces: Acquisition and validation of a new face stimulus set. PLoS ONE, 8(11), 1-7. doi:10.1371/journal.pone.0079131

Dion, K., Berscheid, E., \& Walster, E. (1972). What is beautiful is good. Journal of Personality and Social Psychology, 24(3), 285-90.

Eagly, A. H., Ashmore, R. D., Makhijani, M. G., \& Longo, L. C. (1991). What is beautiful is good, but: A meta-analytic review of research on the physical attractiveness stereotype. Psychological Bulletin, 110(1), 109-128.

Elfenbein, H. A., \& Ambady, N. (2002). Is there an in-group advantage in emotion recognition? Psychological Bulletin, 128(2), 243-249. doi:10.1037/0033-2909.128.2.243

Elfenbein, H. A., \& Ambady, N. (2003). When familiarity breeds accuracy: cultural exposure and facial emotion recognition. Journal of Personality and Social Psychology, 85(2), 276290. doi:10.1037/0022-3514.85.2.276

Ellis, H. C., Thomas, R. L., \& Rodriguez, I. A. (1984). Emotional mood states and memory: elaborative encoding, semantic processing, and cognitive effort. Journal of Experimental Psychology: Learning, Memory, and Cognition, 10(3), 470-82. 
Fink, B., Grammer, K., \& Thornhill, R. (2001). Human (Homo sapiens) facial attractiveness in relation to skin texture and color. Journal of Comparative Psychology, 115(1), 92-99. doi:10.1037//0735-7036.115.1.92

Furnham, A., \& Rawles, R. (1995). Sex differences in the estimation of intelligence. Journal of Social Behavior \& Personality, 10(3), 741-748.

Furnham, A., Reeves, E., \& Budhani, S. (2002). Parents think their sons are brighter than their daughters: sex differences in parental self-estimations and estimations of their children's multiple intelligences. The Journal of Genetic Psychology, 163(1), 24-39. doi:10.1080/00221320209597966

Gurian, M., \& Stevens, K. (2004). With boys and girls in mind. Educational Leadership, 62(3), $21-26$.

Guthrie, R. (1970). Evolution of human threat display organs. Evolutionary Biology, 4, 257-302.

Hayes, A. F. (2013). Introduction to mediation, moderation, and conditional process analysis: A regression-based approach. New York, NY: Guilford.

Hehman, E., Flake, J. K., \& Freeman, J. B. (2015). Static and dynamic facial cues differentially affect the consistency of social evaluations. Personality and Social Psychology Bulletin, 4l(8), 1123-1134. doi:10.1177/0146167215591495

Jones, B. C., Debruine, L. M., Little, A. C., Conway, C. A., \& Feinberg, D. R. (2006). Integrating gaze direction and expression in preferences for attractive faces. Psychological Science, 17(7), 588-91. doi:10.1111/j.1467-9280.2006.01749.x

Kenny, D. A., Horner, C., Kashy, D. A., \& Chu, L. C. (1992). Consensus at zero acquaintance: Replication, behavioral cues, and stability. Journal of Personality and Social Psychology, 
62(1), 88-97.

Kleisner, K., Chvatalova, V., \& Flegr, J. (2014). Perceived intelligence is associated with measured intelligence in men but not women. PloS One, 9(3), e81237. doi:10.1371/journal.pone.0081237

Langlois, J. H., \& Roggman, L. A. (1990). Attractive faces are only average. Psychological Science, 1(2), 115-121. doi:10.1111/j.1467-9280.1990.tb00079.x

Lau, S. (1982). The effect of smiling on person perception. The Journal of Social Psychology, $117,63-67$.

Lim, J., \& Dinges, D. F. (2010). A meta-analysis of the impact of short-term sleep deprivation on cognitive variables. Psychological Bulletin, 136(3), 375-389. doi:10.1037/a0018883

Moore, F. R., Filippou, D., \& Perrett, D. I. (2011). Intelligence and attractiveness in the face: Beyond the attractiveness halo effect. Journal of Evolutionary Psychology, 9(3), 205-217. doi:10.1556/JEP.9.2011.3.2

O’Doherty, J., Winston, J., Critchley, H., Perrett, D. I., Burt, D. M., \& Dolan, R. J. (2003). Beauty in a smile: the role of medial orbitofrontal cortex in facial attractiveness. Neuropsychologia, 41(2), 147-55.

Oginska, H., \& Pokorski, J. (2006). Fatigue and mood correlates of sleep length in three agesocial groups: School children, students, and employees. Chronobiology Iternational, 23(6), 1317-1328. doi:10.1080/07420520601089349

Ohayon, M. M., \& Vecchierini, M. F. (2005). Normative sleep data, cognitive function and daily living activities in older adults in the community. Sleep, 28(8), 981-989.

Olivola, C. Y., Eubanks, D. L., \& Lovelace, J. B. (2014). The many (distinctive) faces of 
leadership: Inferring leadership domain from facial appearance. Leadership Quarterly, 25(5), 817-834. doi:10.1016/j.leaqua.2014.06.002

Olivola, C. Y., Sussman, A. B., Tsetsos, K., Kang, O. E., \& Todorov, A. T. (2012). Republicans prefer republican-looking leaders: Political facial stereotypes predict candidate electoral success among right-leaning voters. Social Psychological and Personality Science, 3(5), 605-613. doi:10.1177/1948550611432770

Oosterhof, N. N., \& Todorov, A. T. (2008). The functional basis of face evaluation. Proceedings of the National Academy of Sciences of the United States of America, 105(32), 1108711092. doi:10.1073/pnas.0805664105

Park, J. H., Schaller, M., \& Van Vugt, M. (2008). Psychology of human kin recognition: Heuristic cues, erroneous inferences, and their implications. Review of General Psychology, 12(3), 215-235. doi:10.1037/1089-2680.12.3.215

Perkinson-gloor, N., Arx, P. H., Brand, S., Holsboer-Trachsler, E., Grob, A., Weber, P., \& Lemola, S. (2015). The role of sleep and the hypothalamic-pituitary-adrenal axis for behavioral and emotional problems in very preterm children during middle childhood. Journal of Psychiatric Research, 60, 141-147. doi:10.1016/j.jpsychires.2014.10.005

Perkinson-Gloor, N., Lemola, S., \& Grob, A. (2013). Sleep duration, positive attitude toward life, and academic achievement: The role of daytime tiredness, behavioral persistence, and school start times. Journal of Adolescence, 36(2), 311-318.

doi:10.1016/j.adolescence.2012.11.008

Perrett, D. I., Burt, D. M., Penton-Voak, I. S., Lee, K. J., Rowland, D. A., \& Edwards, R. (1999). Symmetry and human facial attractiveness. Evolution and Human Behavior, 20(5), 295- 
307. doi:10.1016/S1090-5138(99)00014-8

Perrett, D. I., Lee, K. J., Penton-Voak, I. S., Rowland, D. A., Yoshikawa, S., Burt, D. M., ... Akamatsu, S. (1998). Effects of sexual dimorphism on facial attractiveness. Nature, 394(6696), 884-887.

Pilcher, J. J., \& Huffcutt, A. J. (1996). Effects of sleep deprivation on performance: A metaanalysis. Sleep: Journal of Sleep Research \& Sleep Medicine, 19(4), 318-326.

Quist, M. C., DeBruine, L. M., Little, A. C., \& Jones, B. C. (2012). Integrating social knowledge and physical cues when judging the attractiveness of potential mates. Journal of Experimental Social Psychology, 48(3), 770-773. doi:10.1016/j.jesp.2011.12.018

Re, D. E., \& Perrett, D. I. (2012). Concordant preferences for actual height and facial cues to height. Personality and Individual Differences, 53(7), 901-906. doi:10.1016/j.paid.2012.07.001

Rhodes, G. (2006). The evolutionary psychology of facial beauty. Annual Review of Psychology, 57, 199-226. doi:10.1146/annurev.psych.57.102904.190208

Rosenthal, R., \& Jacobson, L. F. (1968). Teacher expectations for the disadvantaged. Scientific American. doi:10.1038/scientificamerican0468-19

Rule, N. O., \& Ambady, N. (2009). She's got the look: Inferences from female chief executive officers' faces predict their success. Sex Roles, 61(9-10), 644-652. doi:10.1007/s11199-0099658-9

Rule, N. O., \& Ambady, N. (2010). First impressions of the face: Predicting success. Social and Personality Psychology Compass, 8(4), 506-516. doi:10.1111/j.1751-9004.2010.00282.x

Said, C. P., Baron, S. G., \& Todorov, A. T. (2009). Nonlinear amygdala response to face 
trustworthiness: contributions of high and low spatial frequency information. Journal of Cognitive Neuroscience, 21(3), 519-528. doi:10.1162/jocn.2009.21041

Steiger, J. H. (1980). Tests for comparing elements of a correlation matrix. Psychological Bulletin. doi:10.1037/0033-2909.87.2.245

Stephen, I. D., Law Smith, M. J., Stirrat, M. R., \& Perrett, D. I. (2009). Facial skin coloration affects perceived health of human faces. International Journal of Primatology, 30(6), 845857. doi:10.1007/s10764-009-9380-z

Sundelin, T., Lekander, M., Kecklund, G., Van Someren, E. J. W., Olsson, A., \& Axelsson, J. (2013). Cues of fatigue: effects of sleep deprivation on facial appearance. Sleep, 36(9), 1355-1360. doi:10.5665/sleep.2964

Sutherland, C. A. M., Oldmeadow, J. A., Santos, I. M., Towler, J., Burt, D. M., \& Young, A. W. (2013). Social inferences from faces: Ambient images generate a three-dimensional model. Cognition, 127(1), 105-118. doi:10.1016/j.cognition.2012.12.001

Thomas, M., Sing, H., Belenky, G., Holcomb, H., Mayberg, H., Dannals, R., ... Redmond, D. (2000). Neural basis of alertness and cognitive performance impairments during sleepiness: Effects of $24 \mathrm{~h}$ of sleep deprivation on waking human regional brain activity. Journal of Sleep Research, 9(4), 105-118.

Thorndike, E. (1920). A constant error in psychological ratings. Journal of Applied Psychology, $4(1), 25-29$.

Tiddeman, B. P., Burt, D. M., \& Perrett, D. I. (2001). Prototyping and transforming facial textures for perception research. Computer Graphics and Applications, 21(5), 42-50.

Todorov, A. T., Olivola, C. Y., Dotsch, R., \& Mende-Siedlecki, P. (2015). Social attributions 
from faces: Determinants, consequences, accuracy, and functional significance. Annual Review of Psychology, 66(15), 1-27. doi:10.1146/annurev-psych-113011-143831

Todorov, A. T., \& Porter, J. M. (2014). Misleading first impressions: Different for different facial images of the same person. Psychological Science, 25(7), 1404-1417. doi:10.1177/0956797614532474

Walker, M. P. (2009). The role of sleep in cognition and emotion. Annals of the New York Academy of Sciences, 1156, 168-197. doi:10.1111/j.1749-6632.2009.04416.x

Walton, D. (1999). Rethinking the fallacy of hasty generalization. Argumentation, (1), 161-182.

Wickens, C. D., Hutchins, S. D., Laux, L., \& Sebok, a. (2015). The Impact of Sleep Disruption on Complex Cognitive Tasks: A Meta-Analysis. Human Factors: The Journal of the Human Factors and Ergonomics Society. doi:10.1177/0018720815571935

Zebrowitz, L. A., Bronstad, M., \& Lee, H. K. (2007). The contribution of face familiarity to ingroup favoritism and stereotyping. Social Cognition, 25(2), 306-338.

Zebrowitz, L. A., Fellous, J. M., Mignault, A., \& Andreoletti, C. (2003). Trait impressions as overgeneralized responses to adaptively significant facial qualities: evidence from connectionist modeling. Personality and Social Psychology Review, 7(3), 194-215. doi:10.1207/S15327957PSPR0703_01

Zebrowitz, L. A., Kikuchi, M., \& Fellous, J. M. (2010). Facial resemblance to emotions: group differences, impression effects, and race stereotypes. Journal of Personality and Social Psychology, 98(2), 175-189. doi:10.1037/a0017990

Zebrowitz, L. A., \& Montepare, J. M. (1992). Impressions of babyfaced individuals across the life span. Developmental Psychology, 28(6), 1143-1152. 


\section{Figures}

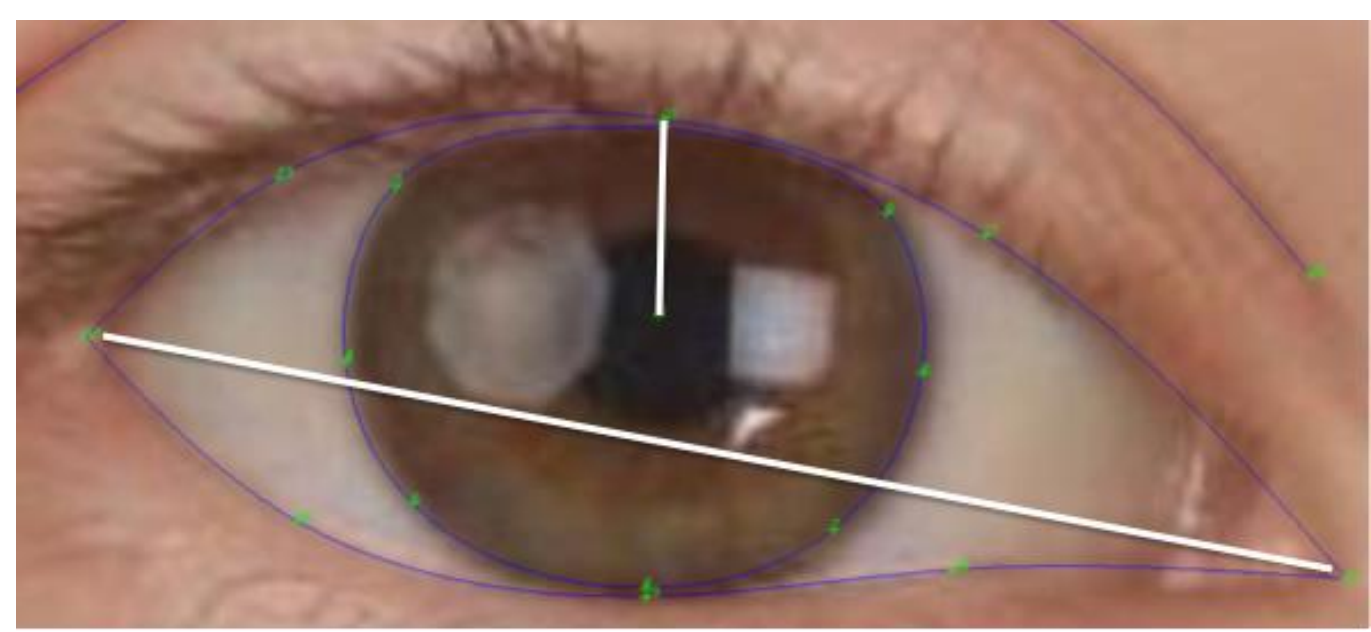

Figure 1. Eyelid-openness calculation. Eyelid-openness was calculated by taking the distance from the center of the pupil to the top eyelid (vertical white line) and dividing it by the width of the eyelid from corner to corner (diagonal white line). 


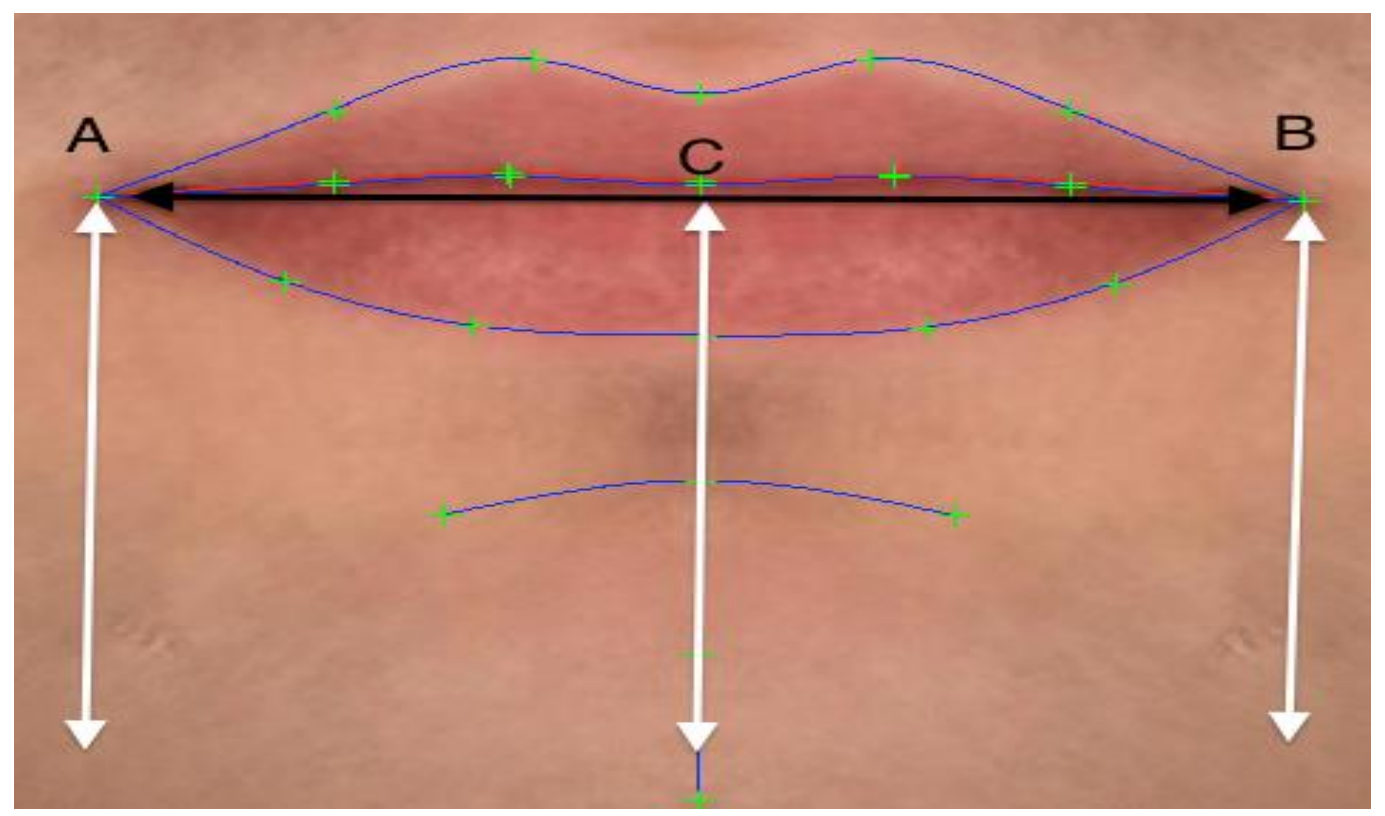

Figure 2. Mouth curvature measure. Example delineation points marked as A (height of left side corner of mouth), B (height of right corner of mouth), and C (height of center of mouth). The height of the center of the mouth (C) was subtracted from the average height of the corners of the mouth (A \& B). This number was then divided by the width of the mouth (distance from A to B). The following formula was used to measure mouth curvature: Mouth corner height relative to center of mouth $=(y A+y B) / 2-y C ;$ Width of mouth $=\operatorname{sqrt}(x A-x B)^{2}+(y A-y B)^{2} ;$ Mouth curvature $=$ Mouth corner height relative to center of mouth / Width of mouth. 


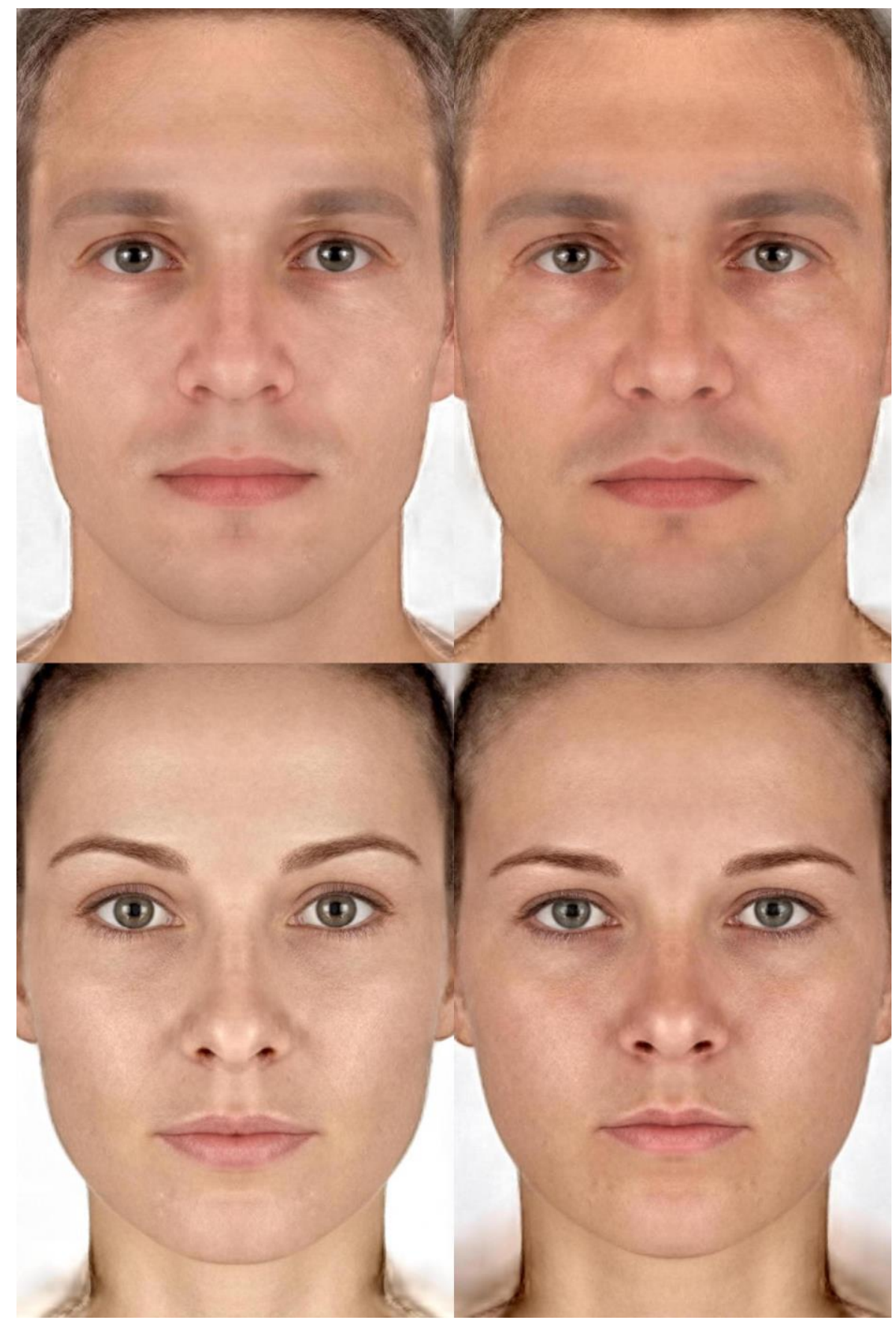

Figure 3. Averaged adult images. High (left) and low (right) perceived intelligence male and female facial averages made symmetrical from Study 1. You may judge for yourself which image in each pair looks more intelligent and more importantly subtle differences in the eyelidopenness and mouth curvature. Objective measures show (slightly) more upturned mouth curvature and eyelid-openness in the high perceived intelligence facial averages images. 
Table 1

Adult Faces: Zero-Order Correlation Matrix

\begin{tabular}{lcccccc}
\hline & Age & Sex & $\begin{array}{c}\text { Perceived } \\
\text { Attractiveness }\end{array}$ & $\begin{array}{c}\text { Perceived } \\
\text { Intelligence }\end{array}$ & $\begin{array}{c}\text { Eyelid- } \\
\text { Openness }\end{array}$ & $\begin{array}{c}\text { Eye } \\
\text { Size }\end{array}$ \\
\hline Sex & .126 & - & & & & \\
Perceived Attractiveness & -.119 & $-.358 * * *$ & - & & & \\
Perceived Intelligence & .059 & $-.373 * * *$ & $.719 * * *$ & - & & \\
Eyelid-Openness & -.076 & .024 & .183 & $.334 * *$ & - & - \\
Eye Size & $-.321 * *$ & -.074 & .191 & .160 & $.603 * * *$ & -.105 \\
Mouth Curvature & $.205 *$ & .040 & .042 & $.237 *$ & -.003 & -.105 \\
\hline
\end{tabular}

$* * * p<.001 . * * p<.01 . * p<.05$.

Note: Sex coded as female $=1$, male $=0 . N=98$. All tests are two-tailed. 
Table 2

Direct and Mediated Effects of Eyelid-Openness, Mouth Curvature, and Gender on Perceived Attractiveness and Perceived Intelligence. (See Figure 4)

\begin{tabular}{lccccc}
\hline Criterion / Predictors & $B$ & $S E$ & $C I$ & $\beta$ & $p$ \\
\hline Perceived Attractiveness & & & & & \\
Eyelid-Openness & 5.32 & 2.58 & $0.21-10.44$ & 0.19 & 0.042 \\
Mouth Curvature & 1.37 & 2.21 & $-3.02-5.76$ & 0.06 & 0.536 \\
Gender & -0.56 & 0.14 & $-0.84--0.27$ & -0.73 & $<0.001$ \\
Perceived Intelligence & & & & & \\
Perceived Attractiveness & 0.44 & 0.05 & $0.34-0.54$ & 0.61 & $<0.001$ \\
Eyelid-Openness & 4.54 & 1.28 & $2.01-7.08$ & 0.23 & $<0.001$ \\
Mouth Curvature & 3.73 & 1.07 & $1.60-5.86$ & 0.22 & $<0.001$ \\
Gender & -0.19 & 0.74 & $-0.33--0.04$ & -0.34 & 0.013 \\
\hline
\end{tabular}

Note: The overall model, as well as the indirect and direct effects of mediating variables, are described in-text. 


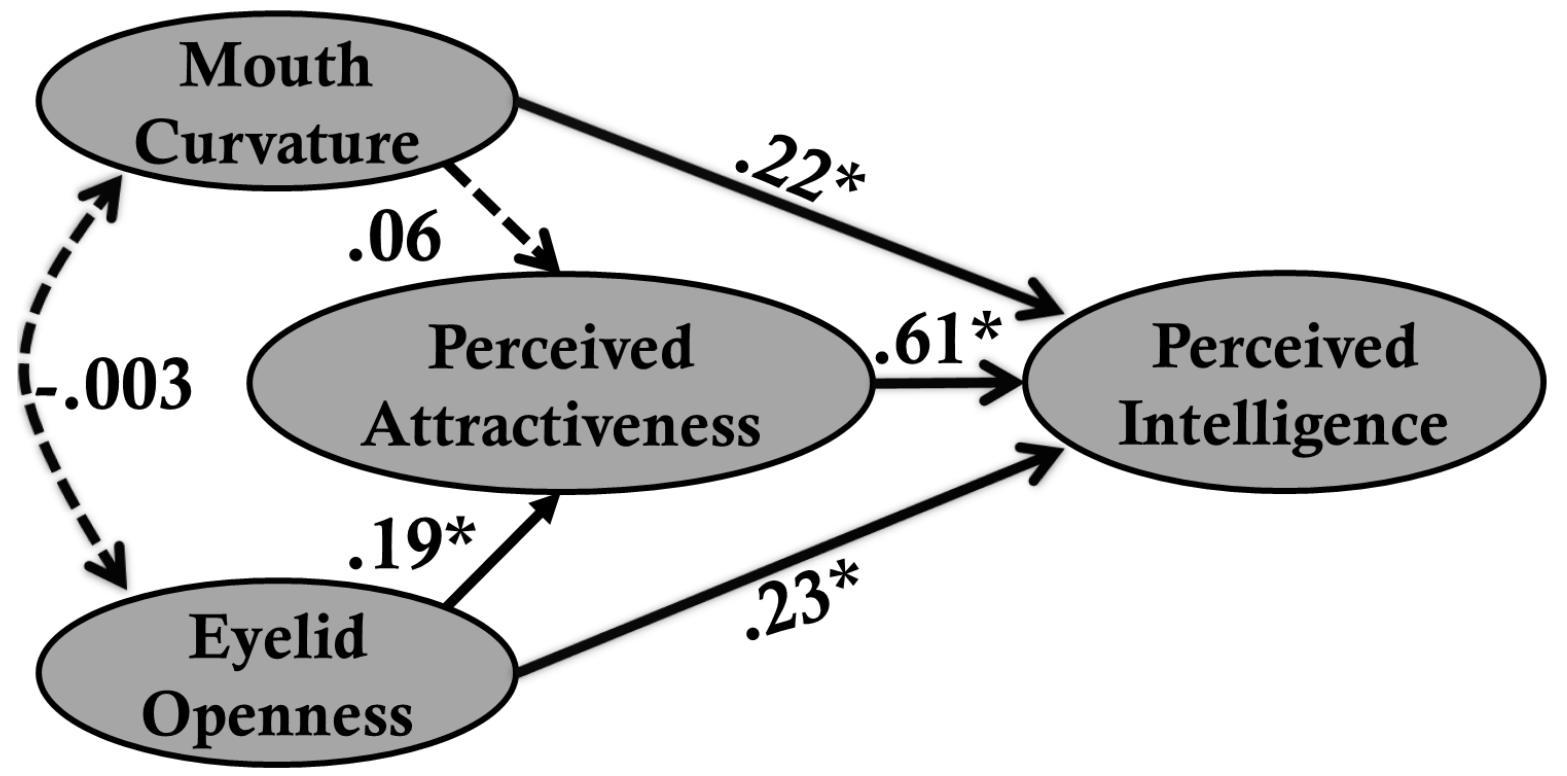

Figure 4. Linear regression model for adult faces. This flow chart shows the independent direct effects $(\beta$ values, $* \mathrm{p}<0.05)$ of upturned mouth curvature, eyelid-openness, and attractiveness on perceived intelligence. Gender of face is included in this model, but not displayed. See results. 
Table 3

Direct and Mediated Effects of Eye Size, Mouth Curvature, and Gender on Perceived Attractiveness and Perceived Intelligence.

\begin{tabular}{lccccc}
\hline Criterion / Predictors & $B$ & $S E$ & $C I$ & $\beta$ & $p$ \\
\hline Perceived Attractiveness & & & & & \\
Eye Size & 66.92 & 36.51 & $-5.55-139.41$ & 0.17 & 0.070 \\
Mouth Curvature & 1.77 & 2.23 & $-2.66-6.20$ & 0.07 & 0.430 \\
Gender & -0.53 & 0.14 & $-0.82--0.25$ & -0.69 & $<0.001$ \\
Perceived Intelligence & & & & & \\
Perceived Attractiveness & 0.47 & 0.05 & $0.36-0.57$ & 0.65 & $<0.001$ \\
Eye Size & 13.62 & 19.02 & $-24.13-51.37$ & 0.05 & 0.476 \\
Mouth Curvature & 3.76 & 1.15 & $1.49-6.04$ & 0.22 & 0.001 \\
Gender & -0.16 & 0.08 & $-0.32--0.01$ & -0.29 & 0.042 \\
\hline
\end{tabular}

Note: The overall model, as well as the indirect and direct effects of mediating variables, are described in-text. 
Table 4

Direct and Mediated Effects of Eye Size, Eyelid-Openness, Mouth Curvature, and Gender on Perceived Attractiveness and Perceived Intelligence.

\begin{tabular}{lccccc}
\hline Criterion / Predictors & $B$ & $S E$ & $C I$ & $\beta$ & $p$ \\
\hline Perceived Attractiveness & & & & & \\
Eye Size & 33.70 & 45.95 & $-57.51-124.91$ & 0.09 & 0.465 \\
Eyelid-Openness & 3.87 & 3.26 & $-2.60-10.34$ & 0.14 & 0.238 \\
Mouth Curvature & 1.57 & 2.23 & $-2.86-6.01$ & 0.07 & 0.482 \\
Gender & -0.55 & 0.14 & $-0.83--0.26$ & -0.71 & $<0.001$ \\
Perceived Intelligence & & & & & \\
Perceived Attractiveness & 0.44 & 0.05 & $0.35-0.54$ & 0.62 & $<0.001$ \\
Eye Size & -37.47 & 22.04 & $-81.24--6.29$ & -0.13 & 0.092 \\
Eyelid-Openness & 6.13 & 1.57 & $3.01-9.25$ & 0.31 & $<0.001$ \\
Mouth Curvature & 3.50 & 1.07 & $1.37-5.62$ & 0.20 & 0.002 \\
Gender & -0.20 & 0.07 & $-0.34--0.05$ & -0.36 & 0.009 \\
\hline
\end{tabular}

Note: The overall model, as well as the indirect and direct effects of mediating variables, are described in-text. 


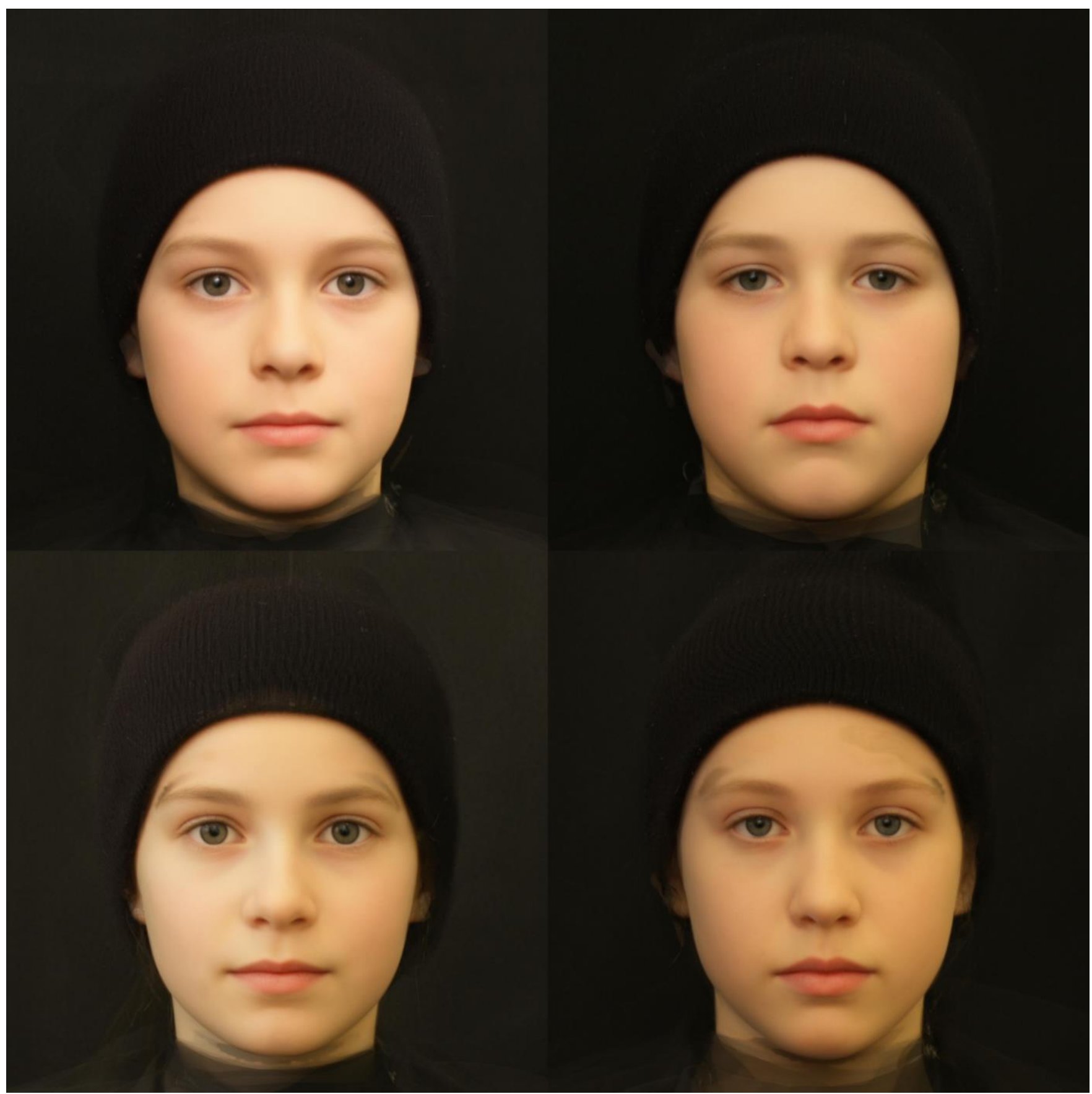

Figure 5. Averaged children images. High (left) and low (right) perceived intelligence female (bottom row) and male (top row) facial averages made symmetrical from Study 2. Note the slightly more upturned mouth curvature and greater eyelid-openness in the high perceived intelligence facial average images. 
Table 5

Children Faces: Zero-Order Correlation Matrix

\begin{tabular}{lccccc}
\hline & Age & Sex & $\begin{array}{c}\text { Perceived } \\
\text { Attractiveness }\end{array}$ & $\begin{array}{c}\text { Perceived } \\
\text { Intelligence }\end{array}$ & $\begin{array}{c}\text { Eyelid- } \\
\text { Openness }\end{array}$ \\
\hline Sex & -.168 & - & & & \\
Perceived Attractiveness & -.022 & $-.276^{* *}$ & - & & \\
Perceived Intelligence & -.006 & $-.268^{*}$ & $.856^{* * *}$ & - & \\
Eyelid-Openness & -.155 & -.057 & $.248^{*}$ & $.391^{* * *}$ & - \\
Mouth Curvature & -.038 & -.118 & .192 & $.311^{* *}$ & .021 \\
\hline
\end{tabular}

$* * * p<.001 . * * p<.01 . * p<.05$.

Note: Sex coded as female $=1$, male $=0 . N=98$. All tests are two-tailed. 
Table 6

Direct and Mediated Effects of Eyelid-Openness, Mouth Curvature, and Gender on Perceived Attractiveness and Perceived Intelligence of Children's Faces. (See Figure 6)

\begin{tabular}{lccccc}
\hline Criterion / Predictors & $B$ & $S E$ & $C I$ & $\beta$ & $p$ \\
\hline Perceived Attractiveness & & & & & \\
Eyelid-Openness & 5.87 & 2.53 & $0.85-10.89$ & 0.23 & 0.023 \\
Mouth Curvature & 2.65 & 1.67 & $-0.67-5.98$ & 0.16 & 0.116 \\
Gender & -0.31 & 0.13 & $-0.56--0.06$ & -0.49 & 0.017 \\
Perceived Intelligence & & & & & \\
Perceived Attractiveness & 0.67 & 0.05 & $0.58-0.77$ & 0.77 & $<0.001$ \\
Eyelid-Openness & 4.35 & 1.14 & $2.09-6.60$ & 0.20 & $<0.001$ \\
Mouth Curvature & 2.29 & 0.74 & $0.82-3.76$ & 0.16 & 0.003 \\
Gender & -0.03 & 0.06 & $-0.14-0.08$ & -0.05 & 0.612 \\
\hline
\end{tabular}

Note: The overall model, as well as the indirect and direct effects of mediating variables, are described in-text. 


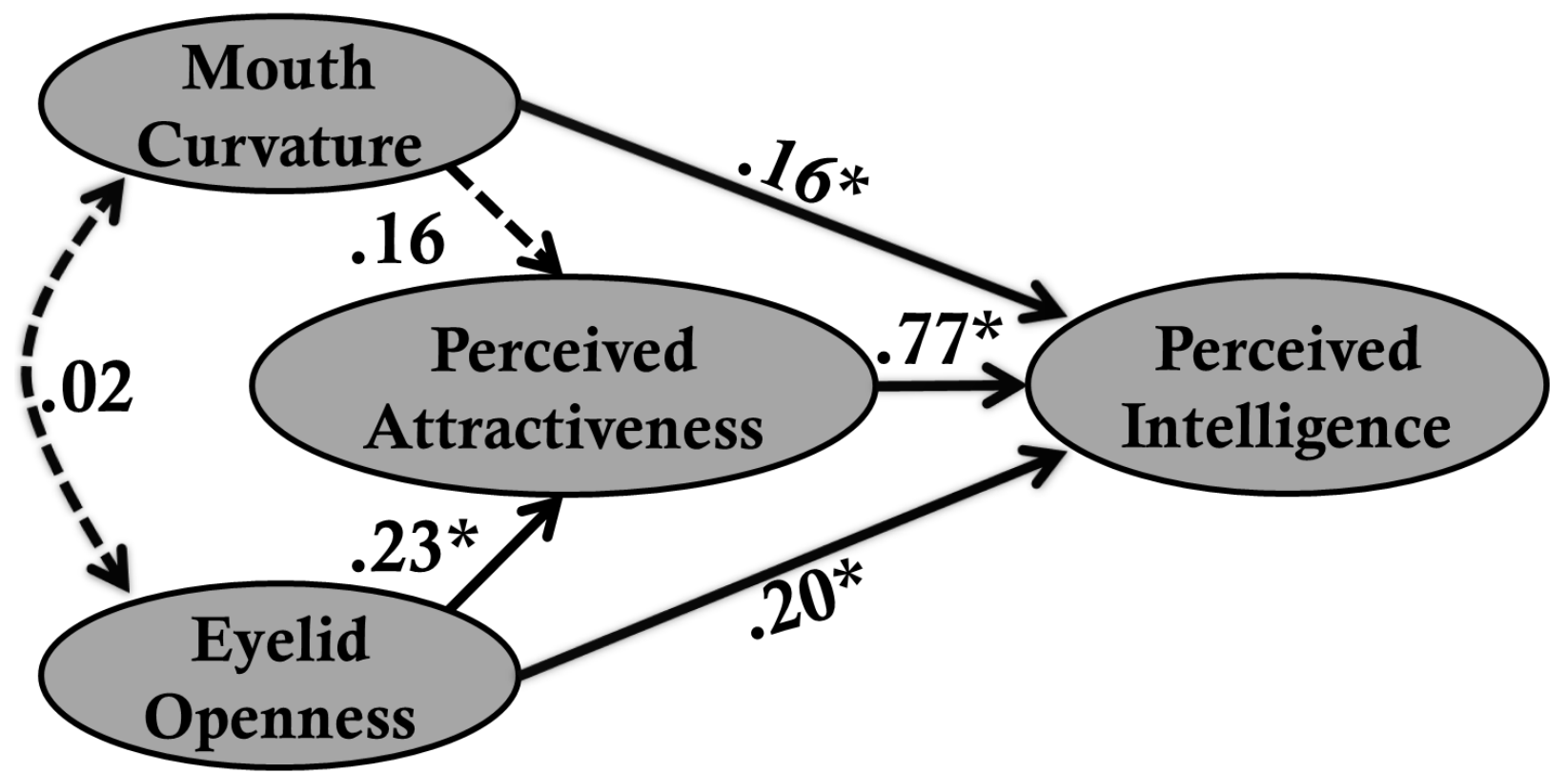

Figure 6. Linear regression model of the perception of children's faces. This flow chart shows the independent direct effects $(\beta$ values, $* \mathrm{p}<0.05)$ of upturned mouth curvature, eyelidopenness, and attractiveness on perceived intelligence. Gender of face is included in this model, but not displayed (See results for details). 


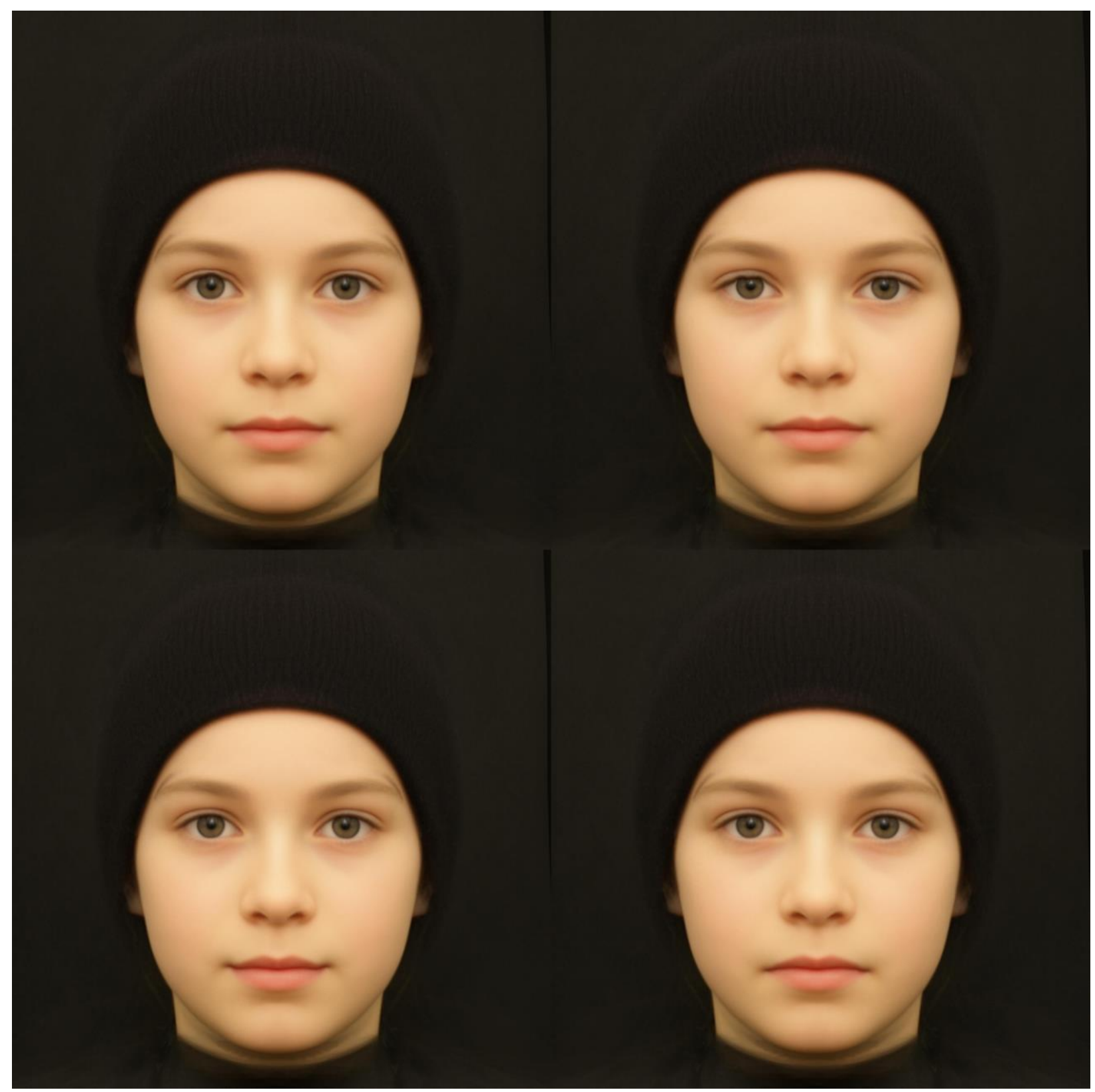

Figure 7. Malleable Facial Cues Transform. An average of all children's faces was manipulated to have increased eyelid-openness (top left) and upturned mouth curvature (bottom left) and decreased eyelid-openness (top right) and downturned mouth curvature (bottom right). The degree of manipulation for this transform was based on a composite image of faces in the set of stimuli with the most and least eyelid-openness and mouth curvature, respectively. 


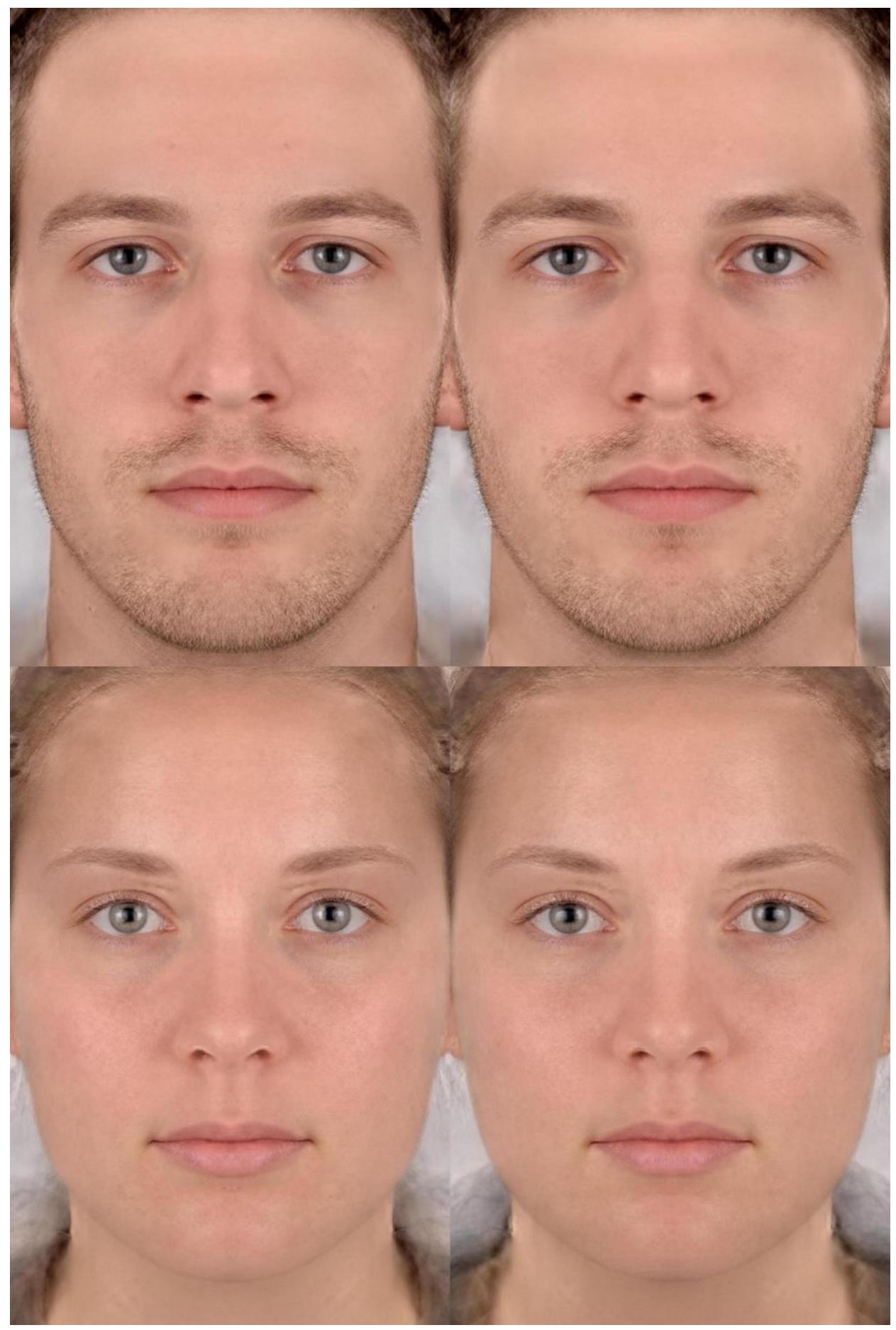

Figure 8. Averaged sleep-restriction images. High (left) and low (right) perceived intelligence male and female facial image averages from Study 4 . There seems to be slightly more upturned mouth curvature and eyelid-openness in the high perceived intelligence facial composite images. 
Table 7

Direct and Mediated Effects of Change in Eyelid-Openness, Change in Mouth Curvature, and Gender on Changes in Perceived Attractiveness and Perceived Intelligence of Adult Faces With and Without Sleep Restriction.(See Figure 9)

\begin{tabular}{lccccc}
\hline Criterion / Predictors & $B$ & $S E$ & $C I$ & $\beta$ & $p$ \\
\hline Perceived Attractiveness & & & & & \\
Eyelid-Openness & 5.44 & 4.20 & $-3.40-14.27$ & 0.28 & 0.212 \\
Mouth Curvature & 2.68 & 1.84 & $-1.18-6.55$ & 0.31 & 0.162 \\
$\quad$ Gender & 0.10 & 0.10 & $-0.12-0.32$ & 0.41 & 0.356 \\
Perceived Intelligence & & & & & \\
Perceived Attractiveness & 0.51 & 0.12 & $0.25-0.77$ & 0.55 & $<0.001$ \\
Eyelid-Openness & 3.57 & 2.27 & $-1.23-8.37$ & 0.20 & 0.135 \\
Mouth Curvature & 3.42 & 1.01 & $1.30-5.54$ & 0.43 & 0.003 \\
Gender & 0.01 & 0.06 & $-0.11-0.13$ & 0.05 & 0.846 \\
\hline
\end{tabular}

Note: The overall model, as well as the indirect and direct effects of mediating variables, are described in-text. 


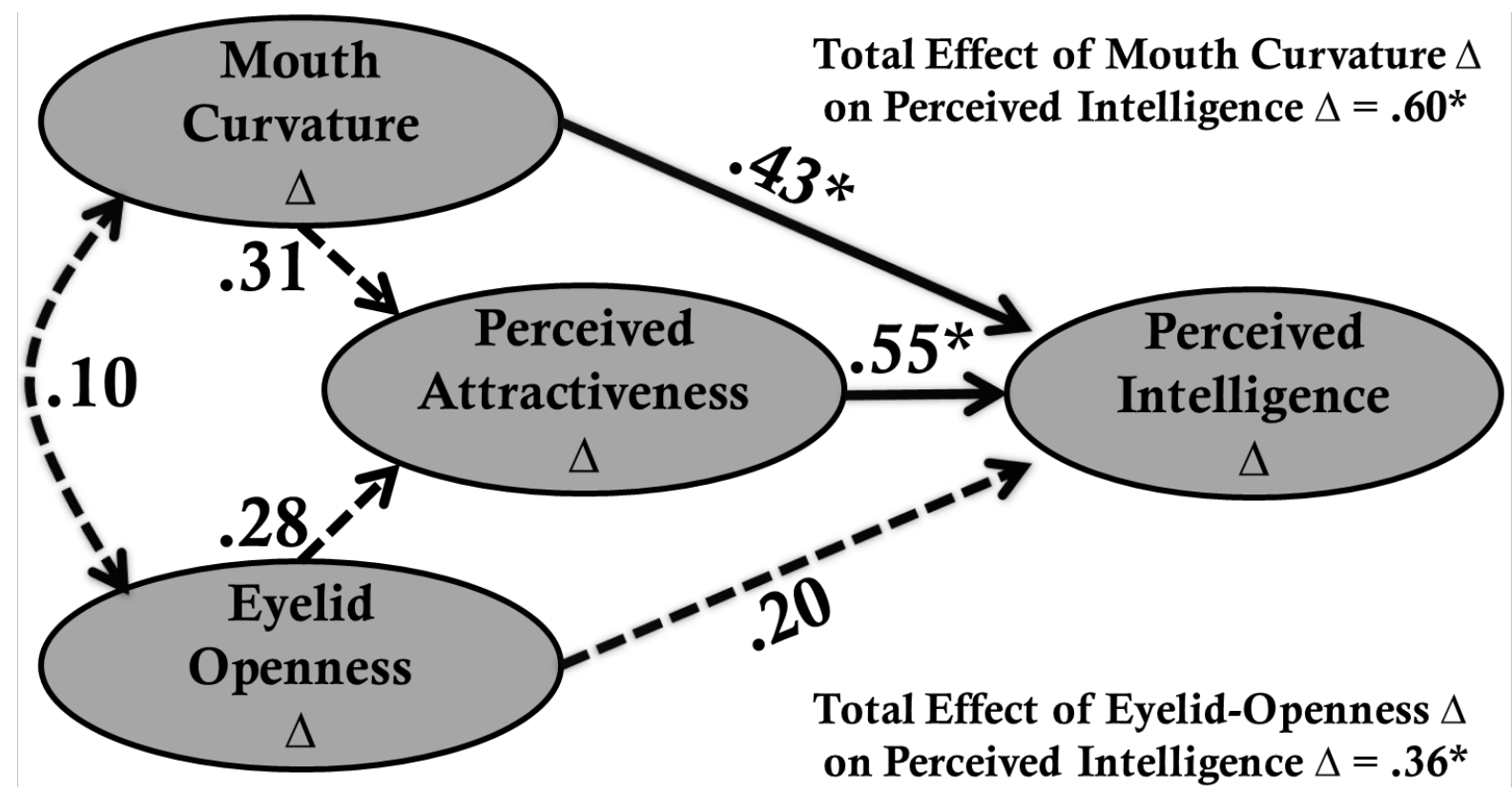

Figure 9. Linear regression model of the perceptual effects of sleep restriction. This flow chart shows the standardized independent direct effects $(\beta$ values, $* \mathrm{p}<0.05)$ of change in mouth curvature and change in eyelid-openness on change in perceived attractiveness and intelligence. Gender of face is included in this model, but not displayed (For details see results). 